\title{
Two-Dimensional Modeling of Ablation and Pyrolysis with Application to Rocket Nozzles
}

\author{
Peter G. Cross* \\ Naval Air Warfare Center Weapons Division, China Lake, CA, 93555 \\ Iain D. Boyd ${ }^{\dagger}$ \\ University of Michigan, Ann Arbor, MI, 48109
}

\begin{abstract}
A new two-dimensional ablation analysis code ("MOPAR-MD") capable of modeling pyrolyzing thermal protection system materials is presented. Favorable agreement with analytical solutions and results from other (one-dimensional) ablation solvers for a wide range of test cases indicates a correct implementation consistent with other codes. This new material response code can be coupled to the "LeMANS" reacting flow solver. New capabilities required for modeling nozzle flow-fields are added to LeMANS, including the Menter BSL and SST turbulence models and a "two gas" method for capturing the thermodynamics of gas-particle flow found in many rocket nozzles. These updated codes are used to perform uncoupled simulations predicting the thermal and ablation response of the "HIPPO" nozzle test case. Radiation is found to have minimal impact on the response of the throat and downstream portions of the rocket nozzle, but remains significant for the motor chamber and upstream portions of the nozzle. Enthalpy conductance and surface recession of the nozzle are found to be quite sensitive to the assumed wall temperature, underscoring the need to perform coupled flow-field / ablation simulations in order to more accurately capture the convective heating environment. All simulations of the HIPPO nozzle predict substantially greater surface recession than measured experimentally. Several potential causes for this discrepancy are identified, many of which could be resolved by performing fully-conjugate, coupled flow-field / ablation simulations.
\end{abstract}

\section{Nomenclature}

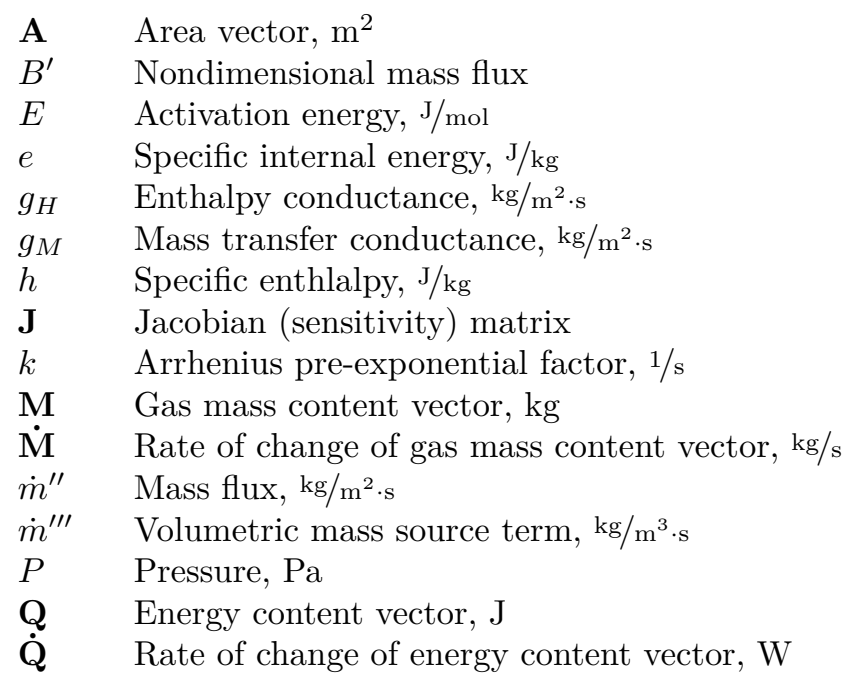

*Aerospace Engineer, Aeromechanics \& Thermal Analysis Branch, 1900 N. Knox Road, M/S 6622, AIAA Senior Member.

$\dagger$ James E. Knott Professor, Department of Aerospace Engineering, 1320 Beale Ave., AIAA Fellow. 


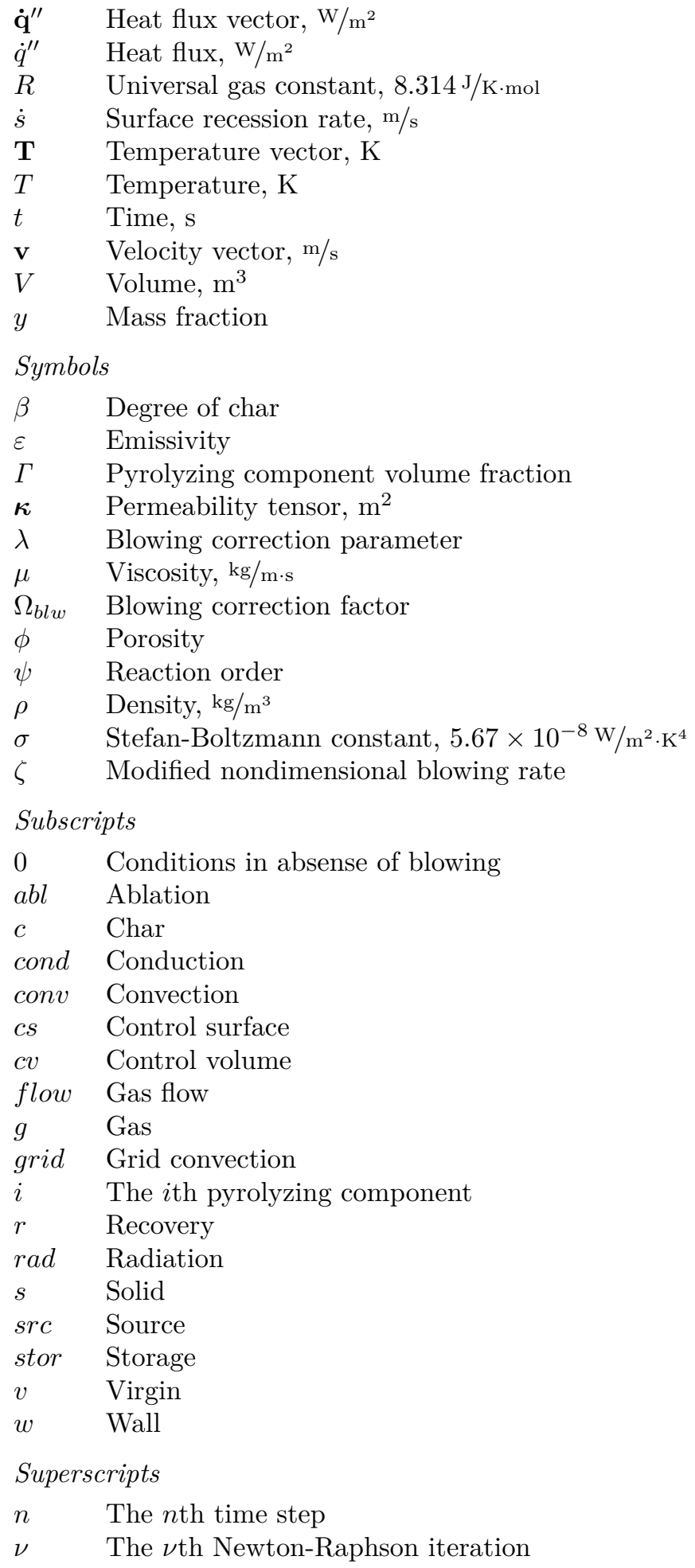

\section{Introduction}

The application for this work is modeling critical rocket nozzle components, which are often manufactured from pyrolyzing, ablating thermal protection system (TPS) materials. Ablation of these materials is a very complex problem involving multiple physical processes and mutual interactions. Historically, the ablation problem has been analyzed in a decoupled manner, relying on empirical correlations to approximate some of the interactions between the flow-field and material response. Because of the insufficiencies associated

$$
2 \text { of } 27
$$


with current ablation analysis methodologies, development of rocket motor components and other thermal management systems relies extensively on costly testing. The research presented here represents a steppingstone towards the establishment of a new engineering methodology that will rigorously capture the strong interactions and dependencies that exist between the reacting flow-field and the ablating material. By basing thermal protection system engineering more firmly on first principles, improved accuracy is anticipated.

Recent work at the University of Rome has investigated the effects of ablation on the flow-field within rocket nozzles. $\frac{1}{3}$ However, this work assumed one-dimensional, steady-state ablation and did not consider the transient thermal response of the TPS material. Multidimensional effects, which can be important near the nozzle throat, were not captured. Researchers at the University of Michigan have performed coupled ablation analyses for external thermal protection systems ${ }^{4}$ - 6 (such as for spacecraft and atmosphere entry vehicles). This work considered the transient response of the TPS material, but only non-charring materials (e.g. graphite) have been modeled assuming two-dimensional heat transfer. Simulations involving pyrolyzing materials have only been performed assuming one-dimensional heat transfer and ablation.

The development and verification of a two-dimensional ablation analysis code capable of modeling pyrolyzing TPS materials (such as carbon phenolic) is presented. First, the relevant governing equations are presented, followed by a discussion of the numerical approached used to solve these equations. Results from verification cases are also presented. Next, new capabilities added to the LeMANS reacting flow-field solver necessary for modeling rocket nozzle flows are discussed, along with associated verification cases. Finally, the material response code and the flow solver are used to investigate the thermal response and ablation of the nozzle for the "HIPPO" motor, a small-scale space shuttle test motor for which there is good experimental data. Multiple comparisons are made to determine the effect and importance of different simulation parameters on the nozzle response.

\section{MOPAR-MD: A Two-Dimensional Material Response Solver}

The material response solver presented here ("MOPAR-MD") builds upon and greatly extends a baseline capability developed by previous researchers at the University of Michigan.5, 7 While this baseline capability did permit two-dimensional ablation analyses, it was restricted in application to non-pyrolyzing TPS materials (e.g. carbon-carbon composite or graphite). However, one strong advantage of this material response code is that it has been coupled to a reacting flow solver ("LeMANS"), which permits tightly-coupled, fully-conjugate simulations of a flow-field and the associated ablation of TPS materials. By adding the capability to model pyrolyzing materials to this material response solver, it will be possible to perform conjugate analysis of ablation within rocket nozzles.

\section{II.A. Governing Equations}

The two-dimensional pyrolysis and ablation capability added to the MOPAR-MD material response code is largely based upon the methods used in the one-dimensional pyrolysis and ablation code developed by Amar et al. 11 and subsequently used in the MOPAR-1D code 12,13 developed at the University of Michigan. The governing equations describing the thermal response of a pyrolyzing ablator on a deforming mesh are:

Mixture energy equation:

$$
\underbrace{\frac{d}{d t} \int_{c v} \rho e d V}_{\text {storage }}=\underbrace{\int_{c s} \rho h \mathbf{v}_{\mathbf{c s}} \cdot d \mathbf{A}}_{\text {grid convection }}-\underbrace{\int_{c s} \phi \rho_{g} h_{g} \mathbf{v}_{\mathbf{g}} \cdot d \mathbf{A}}_{\text {gas flux }}-\underbrace{\int_{c s} \dot{\mathbf{q}}^{\prime \prime} \cdot d \mathbf{A}}_{\text {conduction }}
$$

Solid-phase continuity equation:

$$
\underbrace{\frac{d}{d t} \int_{c v} \rho_{s} d V}_{\text {storage }}=\underbrace{\int_{c s} \rho_{s} \mathbf{v}_{\mathbf{c s}} \cdot d \mathbf{A}}_{\text {grid convection }}+\underbrace{\int_{c v} \dot{m}_{s}^{\prime \prime \prime} d V}_{\text {source }}
$$

Gas-phase continuity equation: 


$$
\underbrace{\frac{d}{d t} \int_{c v} \phi \rho_{g} d V}_{\text {storage }}=\underbrace{\int_{c s} \phi \rho_{g} \mathbf{v}_{\mathbf{c s}} \cdot d \mathbf{A}}_{\text {grid convection }}-\underbrace{\int_{c s} \phi \rho_{g} \mathbf{v}_{\mathbf{g}} \cdot d \mathbf{A}}_{\text {gas flux }}+\underbrace{\int_{c v} \dot{m}_{g}^{\prime \prime \prime} d V}_{\text {source }}
$$

Mass conservation equation:

$$
\underbrace{\int_{c v} \dot{m}_{g}^{\prime \prime \prime} d V}_{\text {source }}+\underbrace{\int_{c v} \dot{m}_{s}^{\prime \prime \prime} d V}_{\text {source }}=0
$$

The velocity of the pyrolysis gases, required in Eqs. (1) and (3), is computed using a form of Darcy's law that can accommodate anisotropic permeability: 14

$$
\mathbf{v}_{\mathbf{g}}=-\frac{\kappa}{\phi \mu} \nabla P
$$

The ideal gas law is used to compute pressure as a function of temperature and pyrolysis gas density.

\section{II.B. Numerical Implementation}

In this material response code, a first-order implicit time integration scheme is employed, along with a second-order spatial discretization scheme based on the control volume finite element method $7,15,17$ and a deformable, unstructured grid. The mesh deformation, energy, solid phase continuity, and gas phase continuity equations are loosely-coupled and solved sequentially in an iterative process each time step. Within each inner iteration, the mesh deformation is solved first, if the surface is receding. Next, the energy equation is solved with an iterative Newton-Raphson scheme. Pyrolysis (thermal decomposition of the TPS material) is then computed by directly solving the solid phase continuity equation. Finally, a NewtonRaphson scheme is used to solve the gas phase continuity equation to obtain the flow of the pyrolysis gases through the porous char. This process is iterated until the maximum change in the temperature solution between inner iterations drops below a threshold value; a threshold value of $\max (\Delta T) \leq 0.1 \mathrm{~K}$ has been found to work well. At this point convergence has been achieved and the solution process advances to the next time step. Typically, only a few $(2-5)$ inner iterations are required in order to achieve convergence at each time step.

\section{II.B.1. Mesh Deformation}

Deformation of the unstructured mesh in response to surface recession is achieved by treating the mesh as a linear elastic solid and solving the elastic solid mechanics equations. Details of this approach can be found in the literature,, 18 and are not repeated here. One advantage of this approach is that the deformation of the mesh is not restricted to follow grid lines, as is the case with some other codes (e.g. TITAN 19 ).

\section{II.B.2. Energy Equation}

The mixture energy equation [Eq. (1)] can be written more compactly as:

$$
\frac{d}{d t} \mathbf{Q}_{\text {stor }}=\dot{\mathbf{Q}}_{\text {grid }}-\dot{\mathbf{Q}}_{f l o w}-\dot{\mathbf{Q}}_{\text {cond }}
$$

Because the material properties are a function of temperature, Eq. (6) represents a system of nonlinear equations, with one equation for each node (control volume) in the mesh. A Newton-Raphson technique is used to solve this system, which requires that each term be linearized in "iteration space". Using the conduction term as an example, this yields:

$$
\dot{\mathbf{Q}}_{\text {cond }}^{\nu}=\dot{\mathbf{Q}}_{\text {cond }}^{\nu}+\mathbf{J}_{\text {cond }}^{\nu} \Delta \mathbf{T}
$$

All terms in Eq. (6) are linearized in a similar fashion. The time derivative is approximated using a backward Euler method:

$$
\begin{gathered}
\frac{d}{d t} \mathbf{Q}_{\text {stor }}=\frac{1}{\Delta t}\left[\mathbf{Q}_{\text {stor }}^{n}-\mathbf{Q}_{\text {stor }}^{n-1}\right]=\frac{1}{\Delta t}\left[\mathbf{Q}_{\text {stor }}^{\nu}+\mathbf{J}_{\text {stor }}^{\nu} \mathbf{\Delta} \mathbf{T}-\mathbf{Q}_{\text {stor }}^{n-1}\right] \\
\frac{4 \text { of } 27}{\text { American Institute of Aeronautics and Astronautics }}
\end{gathered}
$$


which yields first-order implicit time integration.

With these approximations, the following equation is obtained:

$$
\left[\frac{1}{\Delta t} \mathbf{J}_{\text {stor }}^{\nu}+\mathbf{J}_{\text {cond }}^{\nu}-\mathbf{J}_{\text {grid }}^{\nu}+\mathbf{J}_{\text {flow }}^{\nu}\right] \Delta \mathbf{T}=\dot{\mathbf{Q}}_{\text {grid }}^{\nu}-\dot{\mathbf{Q}}_{\text {flow }}^{\nu}-\dot{\mathbf{Q}}_{\text {cond }}^{\nu}-\frac{1}{\Delta t}\left[\mathbf{Q}_{\text {stor }}^{\nu}-\mathbf{Q}_{\text {stor }}^{n-1}\right]
$$

This is an $N \times N$ linear system ( $N$ being the number of nodes in the mesh) that must be solved at each iteration of the Newton-Raphson method. The Generalized Minimum Residual (GMRES) method 20 is used to solve this system; restarting and the ILU(0) preconditioner 21 are used to accelerate convergence of the GMRES solver. The temperature field is updated each Newton-Raphson iteration:

$$
\mathbf{T}^{\nu+1}=\mathbf{T}^{\nu}+\Delta \mathbf{T}
$$

Newton-Raphson iteration is performed until the maximum temperature update falls below a user-specified threshold value. A convergence criteria of $\max (\boldsymbol{\Delta} \mathbf{T}) \leq 10^{-10} \mathrm{~K}$ has been found to work well for the energy equation.

\section{II.B.3. Pyrolysis}

Pyrolysis, or thermal decomposition, of an ablator is described by the solid-phase continuity equation. However, the grid convection term in Eq. (2) greatly complicates the solution of this equation for moving control volumes. An alternative approach is to consider a fixed material element in the domain, for which the solid-phase continuity equation simplifies to:

$$
\underbrace{\frac{d}{d t} \int_{c v} \rho_{s} d V}_{\text {storage }}=\underbrace{\int_{c v} \dot{m}_{s}^{\prime \prime \prime} d V}_{\text {source }}
$$

Traditionally three "species" or "components" are used to describe a pyrolyzing material (with two representing the resin and one representing the reinforcement).22 However, for this work a more generalized modeling approach is taken in which any number of components can be used to describe the pyrolyzing material:

$$
\rho_{s}=\sum_{i} \Gamma_{i} \rho_{i}
$$

Decomposition of each component is assumed to be independent, irreversible, and described by an Arrhenius equation:

$$
\frac{d \rho_{i}}{d t}=-k_{i} \rho_{v_{i}}\left(\frac{\rho_{i}-\rho_{c_{i}}}{\rho_{v_{i}}}\right)^{\psi_{i}} \exp \left(-\frac{E_{i}}{R T}\right)
$$

For each fixed point in the domain, a set of ordinary differential equations describing pyrolysis is obtained. Direct integration of these ODEs is performed implicitly, assuming a linear change in temperature over the duration of a time step. The density history at any point in the domain therefore only depends on the local temperature history. In order to account for the effects of mesh deformation (caused by surface recession), at each time step the solution from the previous time step is interpolated onto the new, deformed mesh.

\section{II.B.4. Porous Flow}

The gas-phase continuity equation describes the flow of the pyrolysis gases through the porous char material. The gas mass source term in the gas-phase continuity equation [Eq. (3)] is linked to the pyrolysis of the material (solid-phase continuity equation [Eq. (2)]) via the mass conservation equation [Eq. (4)]. Thus, an expression for the "instantaneous" gas mass source term can be obtained:

$$
\dot{m}_{g}^{\prime \prime \prime}=\sum_{i} \Gamma_{i} k_{i} \rho_{v_{i}}\left(\frac{\rho_{i}-\rho_{c_{i}}}{\rho_{v_{i}}}\right)^{\psi_{i}} \exp \left(-\frac{E_{i}}{R T}\right)
$$

Amar assumed that this "instantaneous" value of the gas mass source term, as computed at the end of a time step, held constant for the duration of the time step. 9 However, it is observed in this work that this approach can cause a violation of the conservation of mass, since the source term can vary significantly

$$
5 \text { of } 27
$$


across a time step due to the strong dependence on temperature. The mass loss error is observed to decrease in a first-order manner as the time step size is decreased, which is consistent with what would be expected from the first-order implicit time integration scheme. To avoid this mass loss error, an alternative approach is pursued, whereby an "average" volumetric gas mass source term is computed based on the actual change in solid density over the time step:

$$
\dot{m}_{g}^{\prime \prime \prime}=\frac{\rho_{s}^{n-1}-\rho_{s}^{n}}{\Delta t}
$$

With this approach, mass conservation is preserved (all solid mass lost due to pyrolysis becomes gas mass added through the source term).

The gas-phase continuity equation is linearized and solved in a manner similar to that used for the mixture energy equation. The resulting linear system obtained is:

$$
\left[\frac{1}{\Delta t} \mathbf{J}_{\text {stor }}^{\nu}+\mathbf{J}_{\text {cond }}^{\nu}-\mathbf{J}_{\text {grid }}^{\nu}+\mathbf{J}_{\text {flow }}^{\nu}\right] \Delta \boldsymbol{\rho}_{\mathbf{g}}=\dot{\mathbf{M}}_{\text {grid }}^{\nu}-\dot{\mathbf{M}}_{\text {flow }}^{\nu}+\dot{\mathbf{M}}_{\text {src }}^{\nu}-\frac{1}{\Delta t}\left[\mathbf{M}_{\text {stor }}^{\nu}-\mathbf{M}_{\text {stor }}^{n-1}\right]
$$

which is solved using the GMRES method 20 with restarting and the ILU(0) preconditioner. 21 The pyrolysis gas density field is updated each Newton-Raphson iteration:

$$
\rho_{\mathrm{g}}{ }^{\nu+1}=\rho_{\mathrm{g}}^{\nu}+\Delta \rho_{\mathrm{g}}
$$

Newton-Raphson iteration is performed until the maximum pyrolysis gas density update falls below a userspecified threshold value. A convergence criteria of $\max \left(\boldsymbol{\Delta} \boldsymbol{\rho}_{\mathrm{g}}\right) \leq 10^{-5} \mathrm{~kg} / \mathrm{m}^{3}$ has been found to work well for the gas-phase continuity equation. One advantage to this approach for solving the gas-phase continuity equation is that flow of pyrolysis gases is not constrained to follow grid lines, as is the case in some other codes (e.g. TITAN 19 ).

\section{II.B.5. Ablation Boundary Condition}

The net heat flux to an ablating surface is given by:

$$
\dot{q}_{n e t}^{\prime \prime}=\dot{q}_{c o n v}^{\prime \prime}-\dot{q}_{g}^{\prime \prime}-\dot{q}_{c}^{\prime \prime}+\dot{q}_{r a d_{i n}}^{\prime \prime}-\dot{q}_{\text {rad }}^{\prime \prime}
$$

The radiative heat fluxes are not impacted by the ablation process and are easily treated separately. However, the heat fluxes associated with convection, pyrolysis gas loss, and char removal are strongly linked. Considering only these three factors, the heat flux to the surface due to ablation processes is given by:

$$
\dot{q}_{a b l}^{\prime \prime}=\dot{q}_{c o n v}^{\prime \prime}-\dot{q}_{g}^{\prime \prime}-\dot{q}_{c}^{\prime \prime}
$$

In this work the unity Lewis number assumption (mass transfer coefficient and energy transfer coefficient are the same, $g_{M}=g_{H}$ ) is made, which allows the convective heat flux to be modeled as:

$$
\dot{q}_{\text {conv }}^{\prime \prime}=g_{H}\left(h_{r}-h_{w}\right)
$$

where $g_{H}$ is the "enthalpy conductance", an enthalpy-based convection coefficient. (The compound symbol $\rho_{e} u_{e} C_{H}$ is often used to represent enthalpy conductance in the literature.) This enthalpy conductance includes the mitigating effects of blowing:

$$
g_{H}=g_{H_{0}} \Omega_{b l w}
$$

where the blowing correction factor is modeled with the correlation: 23

$$
\begin{gathered}
\Omega_{b l w}=\frac{\zeta}{e^{\zeta}-1} \\
\zeta=\frac{2 \lambda\left(\dot{m}_{g}^{\prime \prime}+\dot{m}_{c}^{\prime \prime}\right)}{g_{H_{0}}}
\end{gathered}
$$

The influence of blowing on laminar boundary layers is modeled with $\lambda=0.5$, while $\lambda=0.4$ is usually used for turbulent boundary layers.

$$
6 \text { of } 27
$$


The heat fluxes associated with pyrolysis gas and char lost from the material due to ablation can be computed as:

$$
\begin{aligned}
& \dot{q}_{g}^{\prime \prime}=\dot{m}_{g}^{\prime \prime} h_{w} \\
& \dot{q}_{c}^{\prime \prime}=\dot{m}_{c}^{\prime \prime} h_{w}
\end{aligned}
$$

Substituting Eqs. (20), (24), and (25) into Eq. (19) yields:

$$
\dot{q}_{a b l}^{\prime \prime}=g_{H}\left(h_{r}-h_{w}\right)-\dot{m}_{g}^{\prime \prime} h_{w}-\dot{m}_{c}^{\prime \prime} h_{w}
$$

Using the definitions for the nondimensional mass fluxes

$$
\begin{aligned}
& B_{g}^{\prime}=\frac{\dot{m}_{g}^{\prime \prime}}{g_{M}}=\frac{\dot{m}_{g}^{\prime \prime}}{g_{H}} \\
& B_{c}^{\prime}=\frac{\dot{m}_{c}^{\prime \prime}}{g_{M}}=\frac{\dot{m}_{c}^{\prime \prime}}{g_{H}}
\end{aligned}
$$

the heat flux to the material surface due to thermochemical convection and ablation can be computed as:

$$
\dot{q}_{a b l}^{\prime \prime}=g_{H}\left[h_{r}-h_{w}\left(1+B_{c}^{\prime}+B_{g}^{\prime}\right)\right]
$$

The enthalpy at the wall $\left(h_{w}\right)$ is the enthalpy of the ablation reaction products, and is a function not only of temperature, but also of the nondimensional mass flux at the boundary of pyrolysis gases and char $\left(B_{g}^{\prime}\right.$ and $B_{c}^{\prime}$ ). Closure of Eq. (29) is achieved by using surface energy balance tables (" $B^{\prime}$ tables") pre-computed by an equilibrium chemistry program, such as ACE. 24 The material response code uses these tables to obtain wall enthalpy and char mass flux as a function of pressure, wall temperature, and nondimensional pyrolysis gas mass flux $\left(B_{g}^{\prime}\right)$. However, one complicating factor is that the pyrolysis gas mass flux leaving the surface is the combination of gas lost due to flow (motion of the gases relative to the material) and the gas stored in material pores lost due to surface recession:

$$
\dot{m}_{g}^{\prime \prime}=\frac{1}{A_{c s}}\left(\int_{c s} \phi \rho_{g} \mathbf{v}_{\mathbf{g}} \cdot d \mathbf{A}+\int_{c s} \phi \rho_{g} \dot{s} d A\right)
$$

Furthermore, surface recession rate $(\dot{s})$ is directly related to $B_{c}^{\prime}$ :

$$
\dot{s}=\frac{\dot{m}_{c}^{\prime \prime}}{\rho_{s}}=\frac{g_{M} B_{c}^{\prime}}{\rho_{s}}=\frac{g_{H} B_{c}^{\prime}}{\rho_{s}}
$$

As a consequence, $B_{g}^{\prime}$ has some dependence on $B_{c}^{\prime}$. Therefore, an iterative procedure is used to determine values for $h_{w}, B_{g}^{\prime}$, and $B_{c}^{\prime}$ that are consistent. With the approach pursued in this material response code, it is not necessary to explicitly relate $\dot{q}_{a b l}^{\prime \prime}$ to the in-depth temperature field when performing the surface energy balance, as is done in other codes (e.g. CMA, 22 ITRAC25). Instead, the heat flux at the surface and the conduction into the material are brought into agreement through the iterative Newton-Raphson method used to solve the energy equation.

\section{II.B.6. Material Properties}

The thermodynamics properties used in Eq. (11) are those for the "mixture", and account for both the solid material and the pyrolysis gases. Mixture density is computed as:

$$
\rho=\phi \rho_{g}+\rho_{s}
$$

while the other properties are computed as mass-weighted averages:

$$
\begin{gathered}
y_{g}=\frac{\phi \rho_{g}}{\rho} \\
\xi=y_{g} \xi_{g}+\left(1-y_{g}\right) \xi_{s}
\end{gathered}
$$

Here $\xi$ is used to represent any property of interest.

$$
7 \text { of } 27
$$


The properties for the solid phase are in turn a function of the degree of char, or the extent of pyrolysis:

$$
\begin{gathered}
\beta=\frac{\rho_{v}-\rho_{s}}{\rho_{v}-\rho_{c}} \\
y_{v}=\frac{\rho_{v}}{\rho_{s}}(1-\beta) \\
\xi_{s}=y_{v} \xi_{s}+\left(1-y_{v}\right) \xi_{c}
\end{gathered}
$$

One exception is the permeability of the solid material, which can vary over several orders of magnitude between the virgin and char states. Experimental data suggest that the permeability varies logarithmically with respect to degree of char. $26-28$ Therefore, the permeability of the pyrolyzing material is modeled as:

$$
\log _{10}(\kappa)=(1-\beta) \log _{10}\left(\kappa_{v}\right)+\beta \log _{10}\left(\kappa_{c}\right)
$$

In MOPAR-MD, all properties are assumed to independently vary linearly within each element in the domain. This is in contrast to some other codes (e.g. CMA, 22 ITRAC, 25 MOPAR-1D 12 , 13) which often combine certain properties into compound pseudo-properties (e.g. " $\rho e^{\prime \prime) . ~}$

\section{II.C. Code Verification}

The pyrolysis, porous flow, and ablation capabilities added to the MOPAR-MD material response solver are verified by performing simulations for a number of test cases that exercise different aspects of the code. By comparing results from MOPAR-MD to results produced by other, accepted ablation codes (ITRAC 25 and MOPAR-1D 12, 13), it is possible to verify that the relevant equations have been correctly implemented into the code. Good agreement is achieved for all test cases considered, indicating that the models describing pyrolysis and ablation have been correctly implemented into MOPAR-MD.

The pyrolysis capability is verified by simulating Case \#1 proposed by Ewing; 25 very good agreement is achieved, with a negligible root mean square (RMS) error of $3 \times 10^{-9}$. Similarly, the porous flow capability is verified by simulating Ewing's Case \#2. Again, excellent agreement is observed with an RMS difference of less than $0.05 \%$ relative to a reference solution. Partial verification of the heat transfer and ablation capabilities is achieved by simulating the Ablation Workshop Test Cases \#1 29 and \#2-1,, 30 for which good agreement is also obtained.

Full verification of the pvrolysis, porous flow, and ablation capabilities is obtained by simulating Ablation Workshop Test Case \#2-2. $\frac{30}{3 n}$ this test case, a $5 \mathrm{~cm}$ thick slab of TACOT (Theoretical Ablative Composite for Open Testing) material ${ }^{31}$ (a hypothetical material model developed as part of the Ablation Workshop activities and somewhat representative of a low-density phenolic material, such as PICA) is subjected to a moderate aeroheating boundary condition. Enthalpy conductance is held constant at $0.3 \mathrm{~kg} / \mathrm{m}^{2} \cdot \mathrm{s} ;$ recovery enthalpy ramps from $0.0 \mathrm{~J} / \mathrm{kg}$ to $1.5 \times 10^{6} \mathrm{~J} / \mathrm{kg}$ over 0.1 seconds. Initial temperature is $298 \mathrm{~K}$; simulation duration is $60 \mathrm{~s}$.

The quasi-1D domain modeled in MOPAR-MD is $5 \mathrm{~mm}$ wide (axial direction). Meshes for MOPAR-MD utilize a topology with stacked anisotropic triangular elements well suited for capturing strong gradients in the wall-normal direction. Four different levels of mesh refinement are considered for this case: 100, 200, 300 , and 600 nodes in the wall-normal (thickness) direction ("coarse", "medium", "fine", and "extra fine", respectively). In order to better capture the strong gradients that form near the heated surface, a stretch ratio of 1.01 is used between subsequent layers of the mesh. All meshes have six nodes in the axial (width) direction. The MOPAR-1D and ITRAC codes use equivalent 1D meshes. Comparisons are made for the "extra fine" mesh, though results from the "fine" mesh are very similar, indicating mesh convergence. A constant time step of $0.01 \mathrm{~s}$ is used for most MOPAR-MD and ITRAC simulations; reducing the time step to $0.001 \mathrm{~s}$ produced negligible change in results. MOPAR-1D simulations use an automatically-computed variable time step. It should be noted that in order to get this test case to solve correctly in ITRAC it is necessary to generate new $B^{\prime}$ tables with many more values of $B_{g}^{\prime}$ than was used in the tables distributed with the TACOT material description. Both MOPAR-MD and MOPAR-1D run satisfactorily with the default $B^{\prime}$ tables, demonstrating reduced sensitivity to the quality of the $B^{\prime}$ tables.

The thermal response of the surface and seven in-depth thermocouple locations is compared in Fig. 1. Very good agreement is obtained between the three codes. RMS difference for the MOPAR-MD thermal response is less than $2.1 \%$ relative to MOPAR-1D and less than $1.7 \%$ relative to ITRAC. Nondimensional 


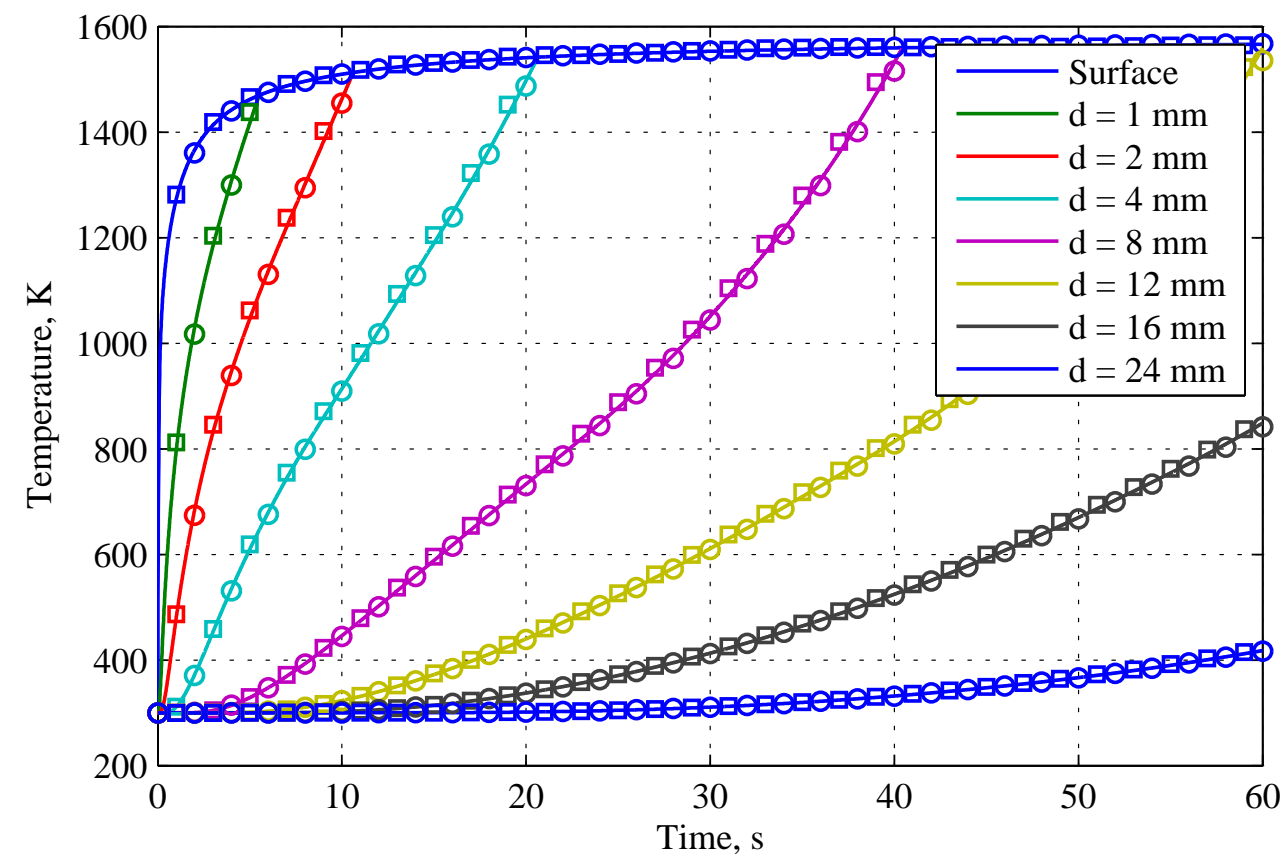

Figure 1. Comparison of surface and in-depth thermal response for Ablation Workshop Test Case \#2-2 as predicted by MOPAR-MD (lines), MOPAR-1D ( $\bigcirc)$, and ITRAC ( $\square)$.

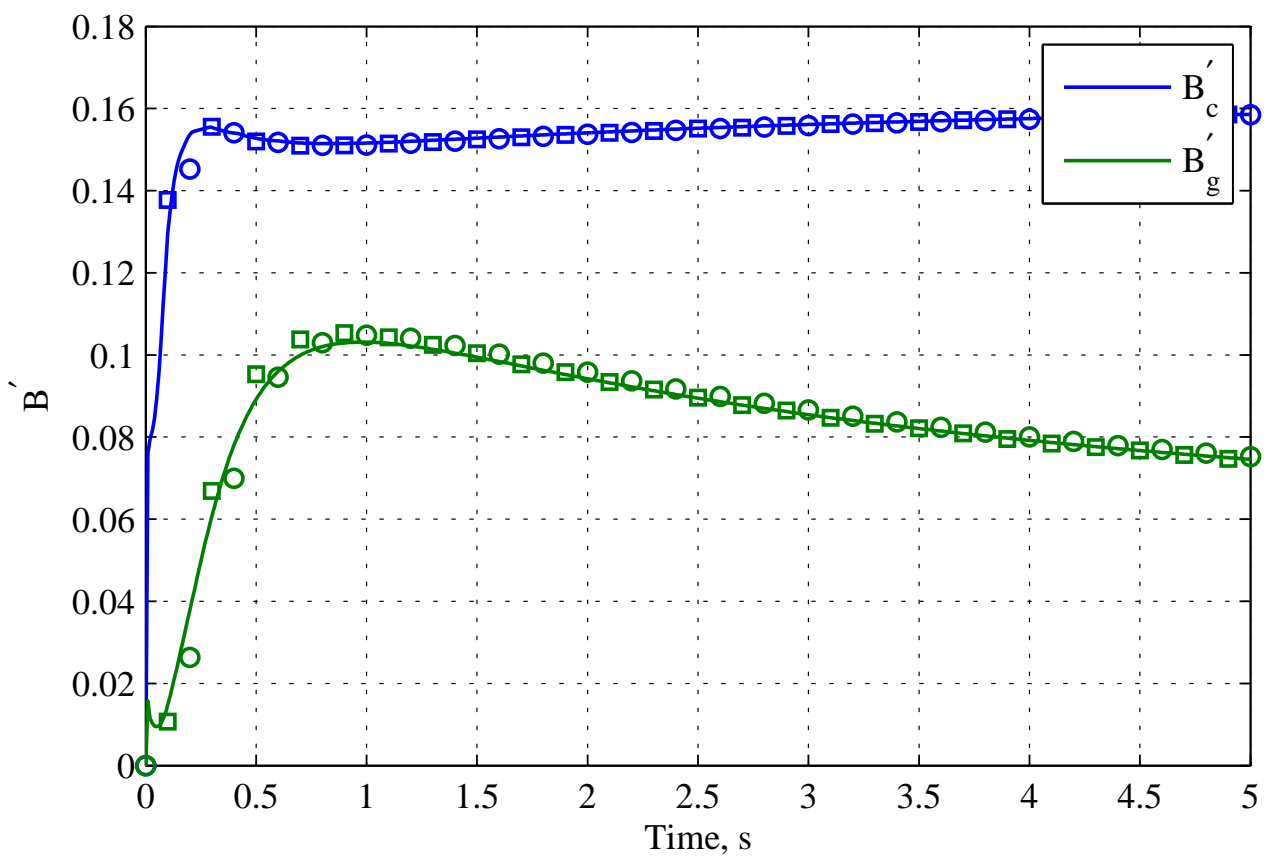

Figure 2. Comparison of nondimensional char mass flux $\left(B_{c}^{\prime}\right)$ and pyrolysis gas mass flux $\left(B_{g}^{\prime}\right)$ for Ablation Workshop Test Case \#2-2 as predicted by MOPAR-MD (lines), MOPAR-1D ( $\bigcirc$ ), and ITRAC ( $\square$ ). 
char mass flux $\left(B_{c}^{\prime}\right)$ and pyrolysis gas mass flux $\left(B_{g}^{\prime}\right)$ are presented as functions of time in Fig. 2. Only data from the initial, transient portion of the simulations are plotted, to accentuate differences between the codes. Excellent agreement is observed here as well, with an RMS difference for $B_{c}^{\prime}$ of $1.1 \%$ relative to MOPAR-1D and $0.3 \%$ relative to ITRAC; for $B_{g}^{\prime}$ the RMS differences are $2.6 \%$ and $1.8 \%$, respectively. Surface recession is predicted by MOPAR-MD to be $1.2 \mathrm{~cm}$; final surface recession values agree within $1.0 \%$. Successful simulation of this test case indicates that all components necessary for modeling the ablation of pyrolyzing materials have been implemented correctly and consistently with other accepted ablation codes.

\section{LeMANS Flow Solver}

The flow solver used in this work is LeMANS, 8 a multi-species, reacting Navier-Stokes solver developed at the University of Michigan. This code was developed for the purpose of studying thermal and chemical nonequilibrium phenomena that occur in two- or three-dimensional, laminar, hypersonic flow-fields. Multiple temperatures are used to model the different energy modes of all species, and different transport property models are available. A modified Steger-Warming Flux Vector Splitting Scheme is used for computing the invisicid fluxes, while a central-difference scheme is used for the viscous fluxes; LeMANS is second-order accurate in space. Steady-state solutions are obtained through a time-marching method. Integration is generally performed using a line-implicit scheme, though a point-implicit scheme is also available. Parallelization is achieved by using MPI and METIS libraries. LeMANS has been coupled to an early version of the MOPAR-MD material response solver that only supports non-pyrolyzing materials. This present work lays the foundation for performing fully-coupled, conjugate simulations with pyrolyzing materials.

\section{III.A. Added Capabilities}

In order to accurately model rocket nozzle flow-fields with the LeMANS flow solver, it was necessary to add models for physical phenomena that are important in rocket nozzle problems. The baseline LeMANS solver only modeled laminar flow, but the flow through rocket nozzles is generally turbulent. Therefore, in this work, two turbulence models are implemented into LeMANS. Solid propellant rockets also have nozzle flows with very high mass fractions of condensed-phase particles. To account for this, a method for modeling the thermodynamics of particle-laden flows is incorporated into the LeMANS flow solver.

\section{III.A.1. Turbulence}

Since nozzle flow-fields are turbulent, in this work the LeMANS flow solver is modified to include an implementation of the Menter BSL and SST k- $\omega$ turbulence models. 32 These closely-related models combine the near-wall accuracy provided by $\mathrm{k}-\omega$ models with the free-stream insensitivity of $\mathrm{k}-\varepsilon$ models. The Menter SST model is a widely-adopted general purpose turbulence model used for a broad range of applications, and has been shown to work particularly well for adverse pressure gradient flows. The BSL model, however, is better suited for favorable pressure gradients (as is the case in rocket nozzles), and is therefore the turbulence model used in this work. A review of the literature shows that both the BSL and SST models can accurately predict convective heating. $33-35$ In order to obtain the most accurate heat transfer predictions, wall functions are not employed. Rather, the turbulence equations are solved through the viscous sublayer to the wall.

The turbulence equations are solved in a loosely-coupled manner, in which the mean flow and turbulence equations are solved sequentially. This commonly-taken approach comes at the cost of potentially slower convergence, but provides the benefits of increased flexibility and greatly simplified implementation. In the present turbulence model implementation, an implicit first-order upwind method is used to compute the inviscid fluxes, while an implicit second-order central-difference scheme is used to compute the viscous fluxes. Source terms are computed using the vorticity-based formulation. An approximate Jacobian is used for the source terms in order to improve the numerical stability of the model. Integration is performed by the LeMANS line-implicit solver. An under-relaxation factor is used to help ensure positivity, while upper and lower bounds are placed on eddy viscosity to prevent certain numerical issues.

The turbulence equations are coupled to the mean flow equations through additional terms in the viscous fluxes. The Reynolds stresses contribute to both the momentum and energy equations; turbulence also enhances the mixture thermal conductivity and species diffusion. It is assumed that turbulence does not affect the source terms in the species conservation equations. Since the goal is not to model combustion, but

10 of 27

American Institute of Aeronautics and Astronautics 
rather to model relatively minor changes in composition for the post-combustion flow, this assumption is believed to be reasonable. Finally, the turbulent kinetic energy is decoupled from the total energy equation, which is valid when the turbulent kinetic energy is small compared to other energy modes.

\section{III.A.2. Gas-Particle Flow}

Condensed-phase particles, predominantly composed of the alumina $\left(\mathrm{Al}_{2} \mathrm{O}_{3}\right)$ formed by combustion of the aluminum fuel in the propellant, are usually present in large quantities in solid rocket nozzle flow. For example, the HIPPO motor ${ }^{36}$ (see section IV) uses a propellant containing $16 \%$ aluminum fuel (by mass); alumina comprises $30 \%$ (by mass) of the combustion products. While particles form a large mass fraction of the flow, they normally occupy only a negligibly small volume fraction of the flow. These particles can either be in the liquid or solid state depending on temperature, and usually start out as liquid droplets in the rocket chamber which then solidify as they cool during their passage through the nozzle.

In this work, the particle-laden combustion gases are modeled using an equilibrium "two gas" method 37 that treats the condensed phase as an additional gas species with special properties. Since the particles occupy a negligibly small volume, the usual compressible flow equations hold without modification, provided that the mixture thermodynamic properties are correctly computed. These thermodynamic properties reduce simply to mass-weighted averages, which is highly compatible with the multi-species framework used in LeMANS. It is, however, necessary to set the gas constant for condensed-phase species to zero (particles do not contribute a partial pressure), which is easily achieved by specifying a very high molecular weight for the condensed-phase species. It is further assumed that the condensed-phase particles do not experience collisions; the condensed phase is treated as an inviscid continuum. This means that the particles do not contribute to transport properties, nor do they diffuse, nor can they serve as collision partners for vibrational relaxation, nor do they participate in reactions.

Underlying this approach is the assumption that the condensed phase particles are in equilibrium with the surrounding gas (same velocity and temperature). Generally speaking, the gas and particles will not be in equilibrium (particles lag the surrounding gas), but to capture these nonequilibrium effects requires detailed information on the size distribution for the condensed-phase particles. Unfortunately, data describing the particle size distribution for rocket nozzle flows are very rare. While the equilibrium two gas approach may not be able to fully resolve all details of the particle-laden flow in rocket nozzles, this method does capture the main thermodynamic effects of the condensed-phase particles in the flow, which is sufficient for this work.

\section{III.B. Code Verification \& Validation}

To ensure that these new capabilities had been correctly implemented into LeMANS, a series of simulations is performed for a wide range of test cases. Comparisons are made to results from other codes, to theoretical values, and/or to experimental data, depending on the test case. A sub-set of these test cases is discussed in the following sections. The good agreement achieved with these test cases indicates that the LeMANS flow solver can be used to accurately predict the turbulent, particle-laden flow-field within rocket nozzles.

\section{III.B.1. Turbulent Heat Transfer}

Verification and validation of the new turbulence model implementation in LeMANS is achieved by simulating a number of test cases available from the NASA turbulence modeling resource website. include flat plates in various Mach number flows, an axisymmetric transonic bump, and a 3D bump. For the sake of brevity, these test cases are not discussed in detail here; good agreement is achieved in all cases, indicating that the turbulence model equations are coded correctly.

Since it is vitally important to accurately predict heat transfer within nozzles, one particularly useful validation case is a nozzle experimentally investigated by Kolozsi. .38 The Kolozsi nozzle was an instrumented converging-diverging nozzle (with a nominal $7.5^{\circ}$ half-angle) through which high-pressure, high-temperature air passed. Wall temperature and heat transfer coefficient data are available for two different conditions: "RunA" $\left(T_{0}=675 \mathrm{~K}, P_{0}=2.55 \mathrm{MPa}\right)$ and "RunB" $\left(T_{0}=622.2 \mathrm{~K}, P_{0}=1.56 \mathrm{MPa}\right)$. The mesh for this nozzle uses a structured topology and has 168 cells in the axial direction and 74 cells in the wall-normal direction. Near-wall cell thickness is $1.27 \times 10^{-6} \mathrm{~m}$ away from the throat dropping to $1.27 \times 10^{-7} \mathrm{~m}$ near the throat. Maximum axial cell dimension is $2.54 \times 10^{-3} \mathrm{~m}$, reducing to $6.34 \times 10^{-4} \mathrm{~m}$ near the throat. Biasing is used

\footnotetext{
${ }^{a}$ Langley Research Center Turbulence Modeling Resource, http://turbmodels.larc.nasa.gov, [cited 2 June 2014].
} 
to produce smooth growth in cell size. This mesh yields wall $Y^{+}$values that remain below one for the length of the nozzle.

The heat transfer coefficient predicted by LeMANS with the two turbulence models is compared to the experimental measurements of Kolozsi in Fig. 3. From this comparison, it is observed that the turbulence model implementation in the LeMANS flow solver can accurately predict heat transfer within nozzles.

\section{III.B.2. Reaction Mechanism}

Verification of the particle-laden flow capability added to LeMANS is achieved by simulating the flow through the HIPPO nozzle (see section IV] and comparing the results for the nozzle centerline to the output from a 1D isentropic expansion reference solution computed using the NASA CEA chemical equilibrium code. $\frac{39}{9}$ For a prescribed chamber pressure of $P_{0}=4.48 \mathrm{MPa}$ the corresponding chamber temperature is computed using CEA to be $T_{0}=3452 \mathrm{~K}$, based on the propellant composition given by Arnold. 36 Three simulations are performed with LeMANS. The first assumes finite-rate chemistry based on the 13 species, 17 reaction mechanism proposed by Troyes. 10 The second assumes equilibrium chemistry, modeled in LeMANS by increasing the forward / backward reaction rates by a factor of 1000. To show the impact that the condensed phase has on the thermodynamics of the nozzle flow, the third simulation excludes the alumina condensed phase, and only considers the 12 gaseous species.

The temperature on the nozzle centerline is plotted as a function of axial position in Fig. 1 . It can be observed that there is generally very good agreement between the CEA reference solution and the two LeMANS solutions that include the condensed phase. However, when the condensed phase is excluded, the temperatures are substantially under-predicted, clearly demonstrating the impact the condensed phase has on the nozzle flow thermodynamics. The temperature profiles for the finite-rate chemistry simulation and the equilibrium chemistry simulation begin to diverge in the downstream portion of the nozzle, indicating that the equilibrium chemistry assumption is not strictly valid for the full nozzle. Some discrepancies exist between the CEA reference solution and the LeMANS results near the exit of the nozzle (axial position greater than $0.13 \mathrm{~m}$ ). The CEA temperatures "plateau" in this region, which is due to the solidification of the alumina particles. However, at this time LeMANS cannot capture the effects of particle phase change, which is why the LeMANS simulations under-predict temperatures by about $300 \mathrm{~K}$ in this area. The LeMANS temperatures do appear to "plateau" in this same general area, but this is due to the presence of an expansion fan. This expansion fan and other multidimensional flow features can be seen in the temperature contour plot in Fig. 5, which clearly shows the departure of real nozzle flow from the usual 1D assumption.

Species mass fractions on the nozzle centerline are plotted in Fig. 6. The results from the LeMANS simulation assuming equilibrium chemistry agrees quite well with the CEA reference solution, until an axial position of approximately $0.13 \mathrm{~m}$ (where the temperature solutions diverge, as previously discussed). The finite-rate chemistry LeMANS simulation agrees well with the equilibrium results up until an axial position of $0.075 \mathrm{~m}$, at which point the flow appears to become frozen.

These simulations serve to verify that the particle-laden flow capability and the reaction mechanism are working correctly. Additionally, these simulations reveal the importance of capturing the thermodynamics of the condensed phase, and the need for using finite-rate chemistry and a multidimensional flow solver to accurately predict nozzle flow.

\section{Analysis of HIPPO Nozzle}

The "HIPPO" nozzle 36 is selected as a test case for demonstrating two-dimensional modeling of pyrolysis and ablation. The HIPPO motor is a sub-scale $(6.35 \mathrm{~cm}$ diameter throat) space shuttle solid rocket test motor. Four different motors were fired, with each nozzle manufactured from a different carbon phenolic material. Nozzle \#1 is chosen for consideration in this study. Experimental data for this test case are quite comprehensive, with surface recession and char depth being provided as a function of axial position for eight circumferential stations.

\section{IV.A. Model}

A geometrical description of the HIPPO nozzle was provided by Arnold ${ }^{36}$ only for the throat and downstream portions of the nozzle, as shown in Fig. 5. No information was provided describing the nozzle contour more than about $0.13 \mathrm{~m}$ upstream of the throat. Therefore, the flow domain in this upstream region must be 


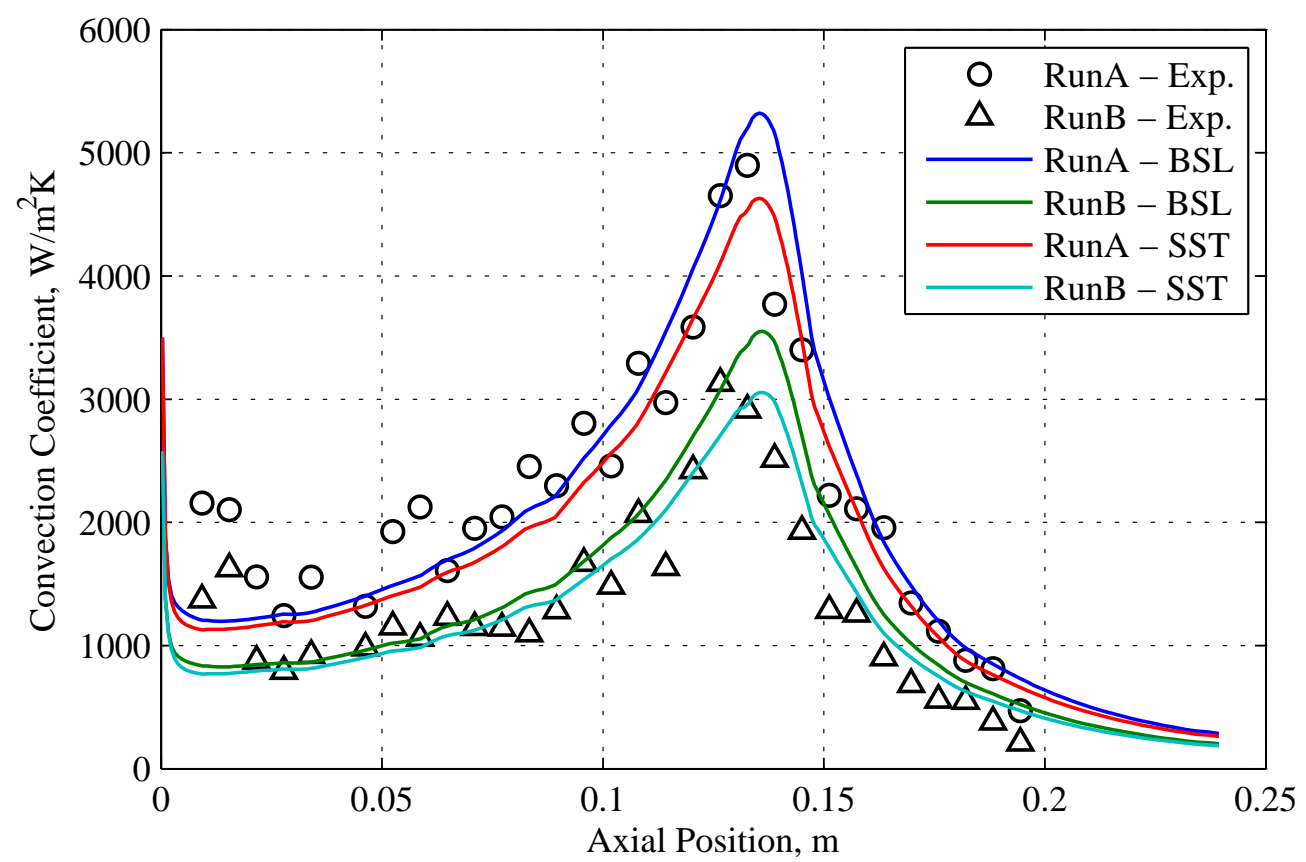

Figure 3. Comparison of convection coefficient computed by LeMANS with Menter BSL and SST turbulence models to the experimental measurements of Kolozsi.

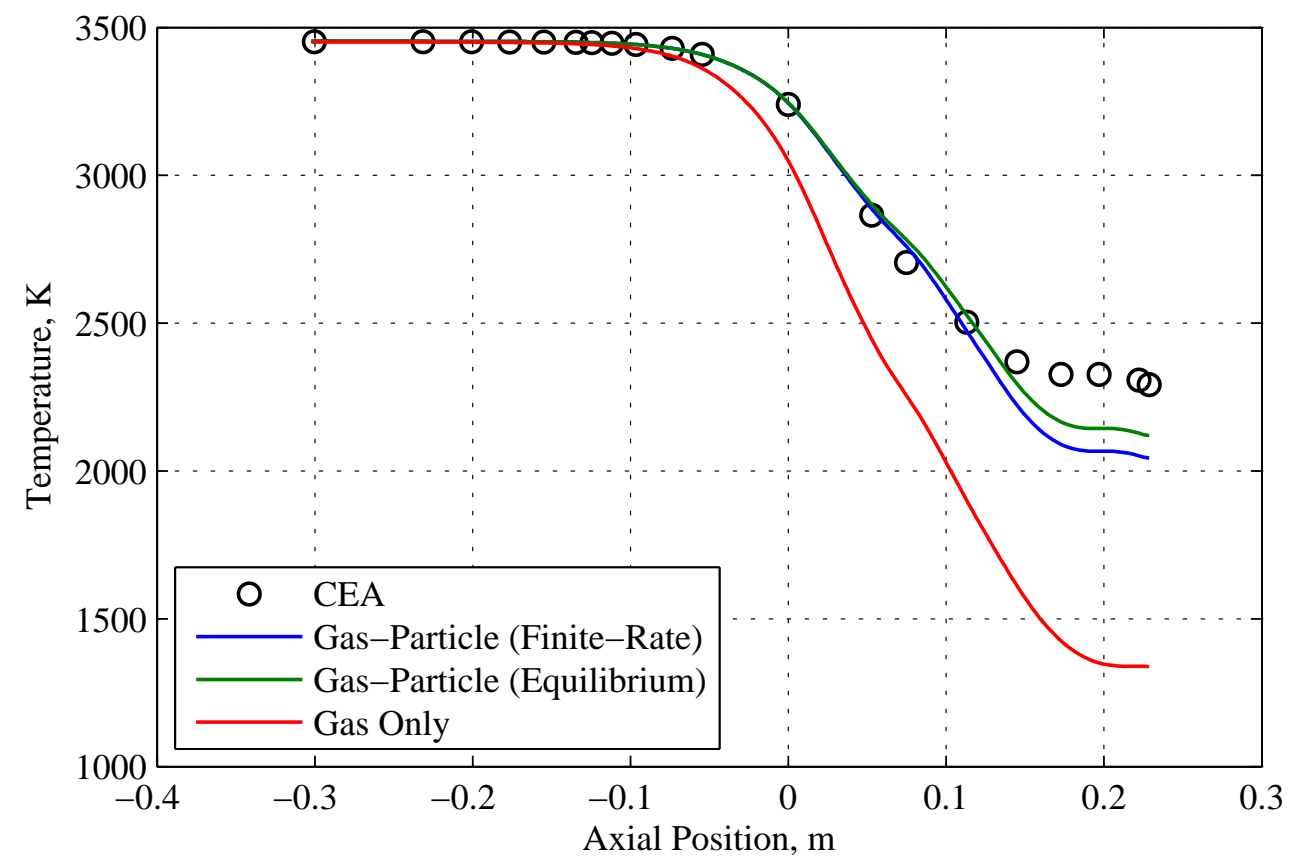

Figure 4. Nozzle centerline temperature as computed with LeMANS compared to the CEA reference solution. 
T[K]: 200021002200230024002500260027002800290030003100320033003400

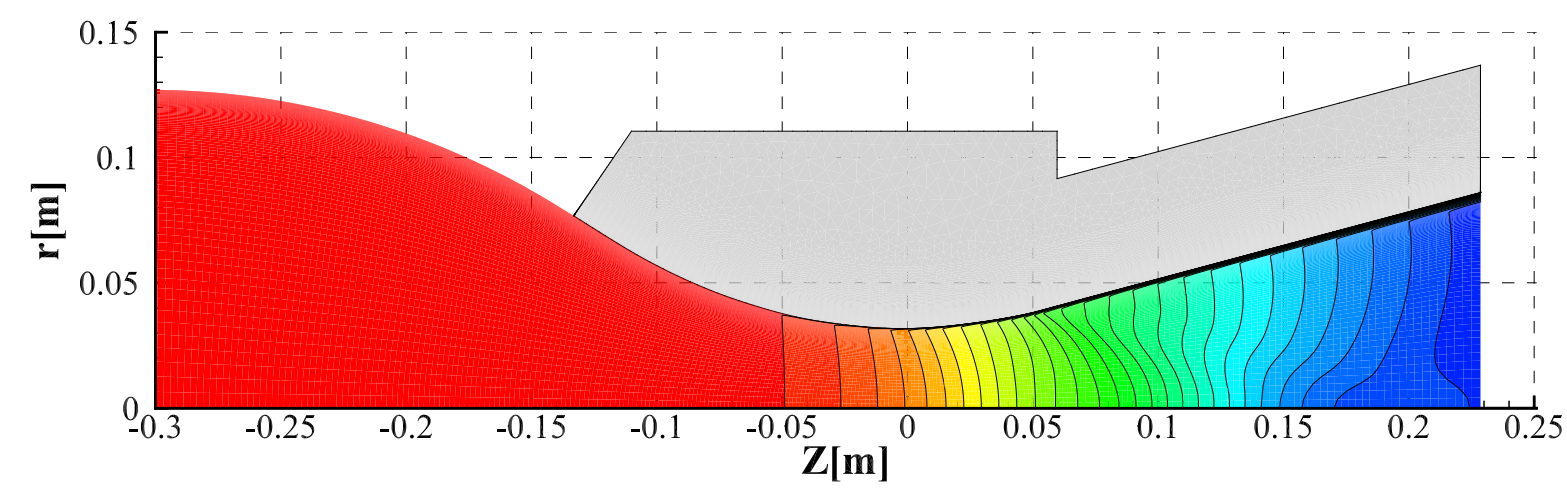

Figure 5. Temperature solution for the HIPPO nozzle as computed with LeMANS assuming finite-rate chemistry. Material response domain is indicated by gray region.

assumed, as shown in Fig. 5, and is most likely different from the actual nozzle geometry. However, it is believed that this difference will not have any impact on the solution at the throat or downstream regions of the nozzle. Additionally, a steel shell surrounded the carbon phenolic material, but this is excluded in the thermal model and is replaced with an adiabatic boundary condition. This will have no impact on the results, as there is no change in temperature observed on this boundary.

The mesh for the flow domain has 150 cells in the wall-normal direction and 267 cells in the axial direction, for a total of 40,050 cells. Near wall cell thickness is $2.54 \times 10^{-6} \mathrm{~m}$, decreasing to $6.35 \times 10^{-7} \mathrm{~m}$ at the throat. The axial dimension of the cells is approximately $2.54 \times 10^{-3} \mathrm{~m}$, decreasing to $1.27 \times 10^{-3} \mathrm{~m}$ near the throat. Biasing is used in order to obtain smooth meshes. Wall $Y^{+}$values remain below one for the length of the nozzle with this mesh.

For the solid domain, a $2.54 \mathrm{~cm}$ thick layer of stacked anisotropic triangular elements is generated adjacent to the ablating boundary. 80 layers of elements are used in this region, with a near-wall thickness of $2.54 \times 10^{-5} \mathrm{~m}$ and a growth rate of 1.05 . Axial dimension of the mesh elements is about $3.2 \times 10^{-3} \mathrm{~m}$. The remainder of the domain is filled with approximately isotropic triangular elements. The final mesh contains 20,235 elements and 10,292 nodes. Comparisons of results computed with this mesh to results produced by a much more refined, quasi-1D mesh for the throat region suggests that further mesh refinement would have minimal impact on the thermal response and surface recession of the nozzle. Simulations use a variable time step according to the schedule presented in Table 1, which makes it possible to capture early transient effects while not using an unnecessarily small time step later in the simulation.

Table 1. Time step schedule used for HIPPO nozzle simulations.

\begin{tabular}{cc}
\hline Time, s & $\Delta t, \mathrm{~s}$ \\
\hline 0.0 & $1.0 \times 10^{-6}$ \\
$1.0 \times 10^{-5}$ & $1.0 \times 10^{-5}$ \\
$1.0 \times 10^{-4}$ & $1.0 \times 10^{-4}$ \\
0.001 & 0.001 \\
0.5 & 0.001 \\
1.0 & 0.1 \\
\hline
\end{tabular}

The HIPPO nozzle was manufactured from MX4926 carbon phenolic material; most properties describing this material are provided by Arnold. $\frac{36}{}$ Additional information describing the orthotropic thermal conductivity is provided by Schaefer. $\underline{11}$ Porosity data are taken from Amar, 9 while permeability data come from McManus. 28 The material is in the $90^{\circ}$ orientation; the plies are oriented perpendicular to the nozzle axis. This means that the thermal conductivity and permeability are larger normal to the axis than along the axis. $B^{\prime}$ tables are computed using ACE. 24 


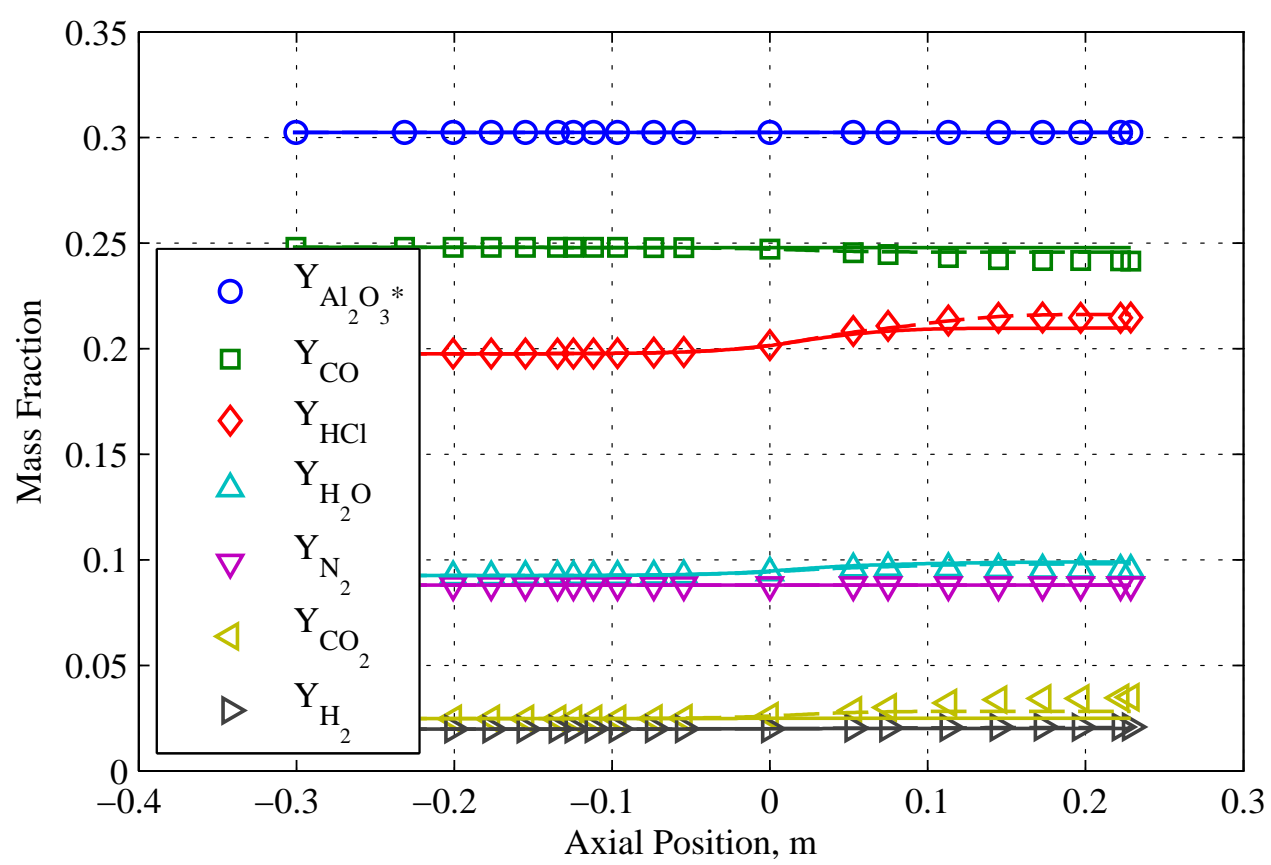

(a) Major species.

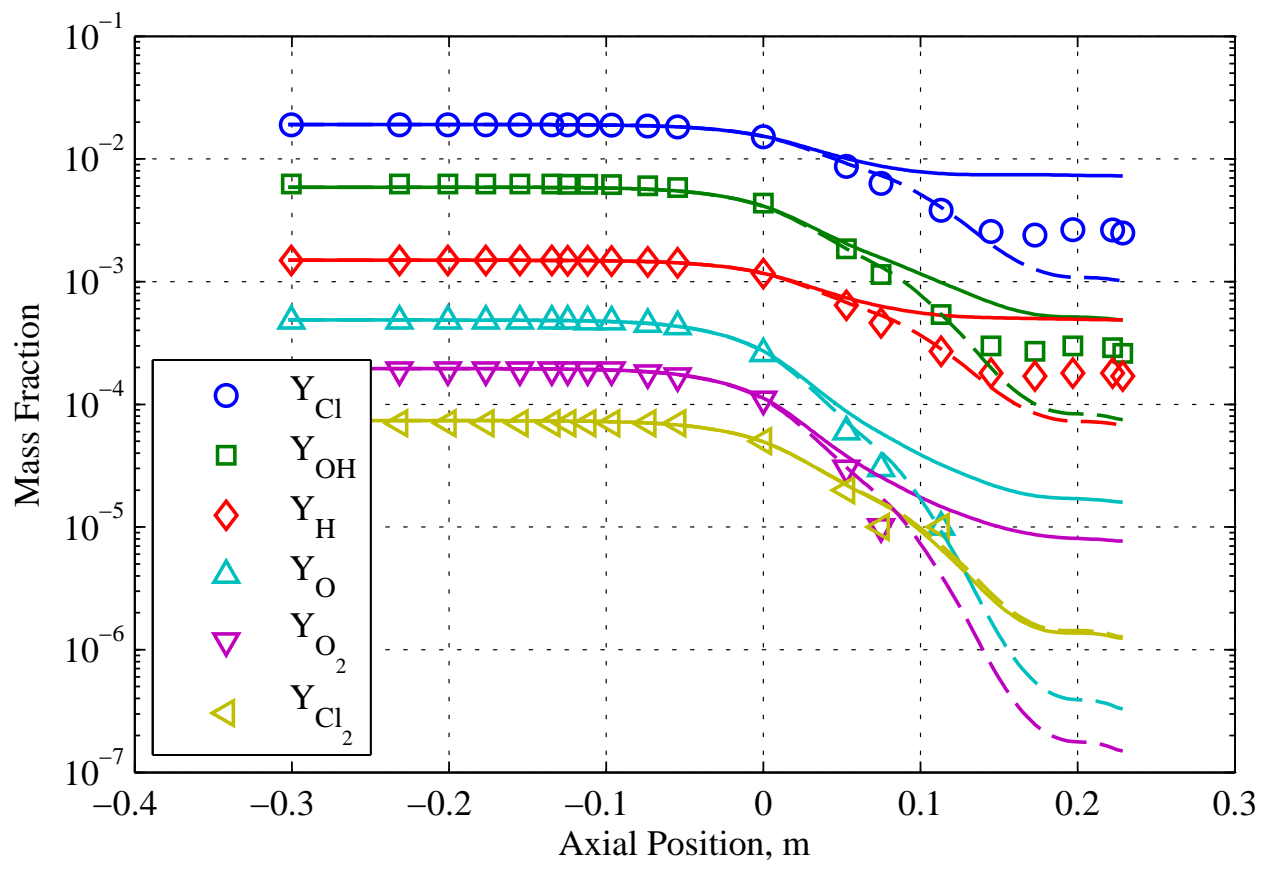

(b) Minor species.

Figure 6. Species mass fractions on the centerline of the HIPPO nozzle, plotted as a function of axial position. Symbols represent the CEA reference solution, solid lines mark LeMANS results assuming finite-rate chemistry, and dashed lines indicate LeMANS results assuming equilibrium chemistry. 


\section{IV.B. Boundary Conditions}

The duration of operation for the HIPPO nozzle was $29.5 \mathrm{~s}$; the experimental pressure trace provided by Arnold 36 is plotted in Fig. 7. This pressure trace is closely approximated for the analysis by using seven discrete pressures. Boundary condition computations are performed for each discrete pressure, and linear interpolation is used to obtain boundary condition values at intermediate pressures. Details of the boundary condition calculations are given in the sections that follow.

\section{IV.B.1. Radiation}

Rockets motors using aluminized propellants produce combustion gases that are highly radiative (due to the presence of alumina particles). Modeling this "stream radiation" requires that stream emissivity and stream temperature be supplied to the material response code. These stream properties are computed using the Nozzle Aerothermochemistry (NAT) code. 42 Stream temperature is taken to be that computed by a 1D isentropic expansion calculation performed by the ACE module included in NAT. The correlation:

$$
\varepsilon_{\text {stream }}=1-\exp \left(-0.808 \frac{n}{16} \rho D\right)
$$

is used to compute the stream emissivity. ${ }^{43}$ Here $n$ is the percent of aluminum in the propellant, $\rho$ is the local density of the combustion products (in units of $\mathrm{lbm} / \mathrm{ft}^{3}$ ), and $D$ is the local diameter of the nozzle (in units of inches).

The stream (combustion gases) is modeled as a 2D slab adjacent to the nozzle surface. Radiation exchange between the gases and the surface can therefore be computed as:

$$
q_{\text {rad }}^{\prime \prime}=\sigma \varepsilon_{\text {eff }}\left(T_{\text {stream }}^{4}-T_{\text {wall }}^{4}\right)
$$

where:

$$
\varepsilon_{\text {eff }}=\frac{1}{\frac{1}{\varepsilon_{\text {stream }}}+\frac{1}{\varepsilon_{\text {wall }}}-1}
$$

is the effective emissivity describing radiative exchange between two gray, infinite, parallel plates.

\section{IV.B.2. Convection}

The convection boundary conditions (recovery enthalpy and enthalpy conductance) are computed using two different methods for comparison. The first method utilizes the NAT code $\underline{42}$ to perform a 1D isentropic expansion calculation to obtain the edge conditions and recovery enthalpy, followed by an integral solution of the boundary layer to obtain the enthalpy conductance. The second method uses the LeMANS flow solver to perform simulations from which the recovery enthalpy and enthalpy conductance can be obtained.

In order to perform these convection boundary condition calculations, it is necessary to estimate the temperature of the nozzle wall adjacent to the flow. To explore the sensitivity of the enthalpy conductance to the assumed wall temperature, a study is performed in which three different wall temperature profiles are considered. The profiles correspond to a wall temperature offset of $100 \mathrm{~K}, 500 \mathrm{~K}$, and $1000 \mathrm{~K}$ below the local recovery temperature; chamber pressure is $4.48 \mathrm{MPa}$. Results from this study are presented in Fig. 8. Several observations can be readily made. The first is that the values for enthalpy conductance as predicted by NAT are higher than those predicted by LeMANS. The exact cause for this discrepancy is unclear, but is almost certainly due to the widely different methods employed. It is expected that the results from LeMANS are more accurate, since the underlying flow physics are being modeled with more rigor. NAT simulations of the Kolozsi nozzle (see section III.B.1) also over-predict the convection coefficient; LeMANS provides better agreement. Another observation that can be made is that the assumed wall temperature has a significant impact on the magnitude of the predicted enthalpy conductance; lower wall temperatures yield higher enthalpy conductance values. It is also observed that the enthalpy conductance as computed with LeMANS is more sensitive to wall temperature than the NAT results. Again, the exact cause for this discrepancy is not clear, but is most likely due to LeMANS capturing more details of the nozzle flow-field.

The main conclusions that can be made from this study are that wall temperature has a strong impact on enthalpy conductance, and that accurate wall temperature values are required in order to obtain accurate enthalpy conductance values. This underscores the importance of being able to perform fully-coupled, conjugate analyses of the nozzle flow-field and ablation response.

16 of 27 


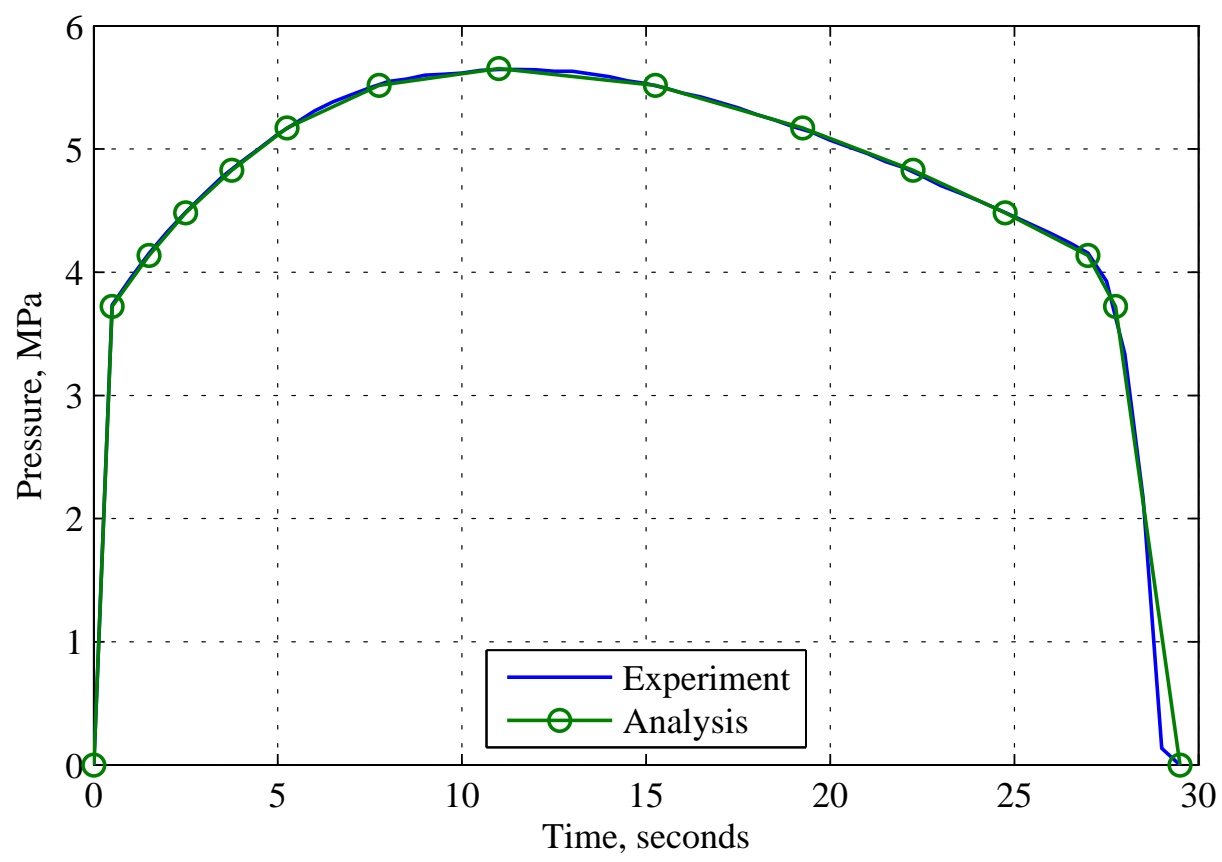

Figure 7. Pressure trace for the HIPPO motor, comparing the discrete pressure trace used in the analysis to the experimentally measured pressure trace.

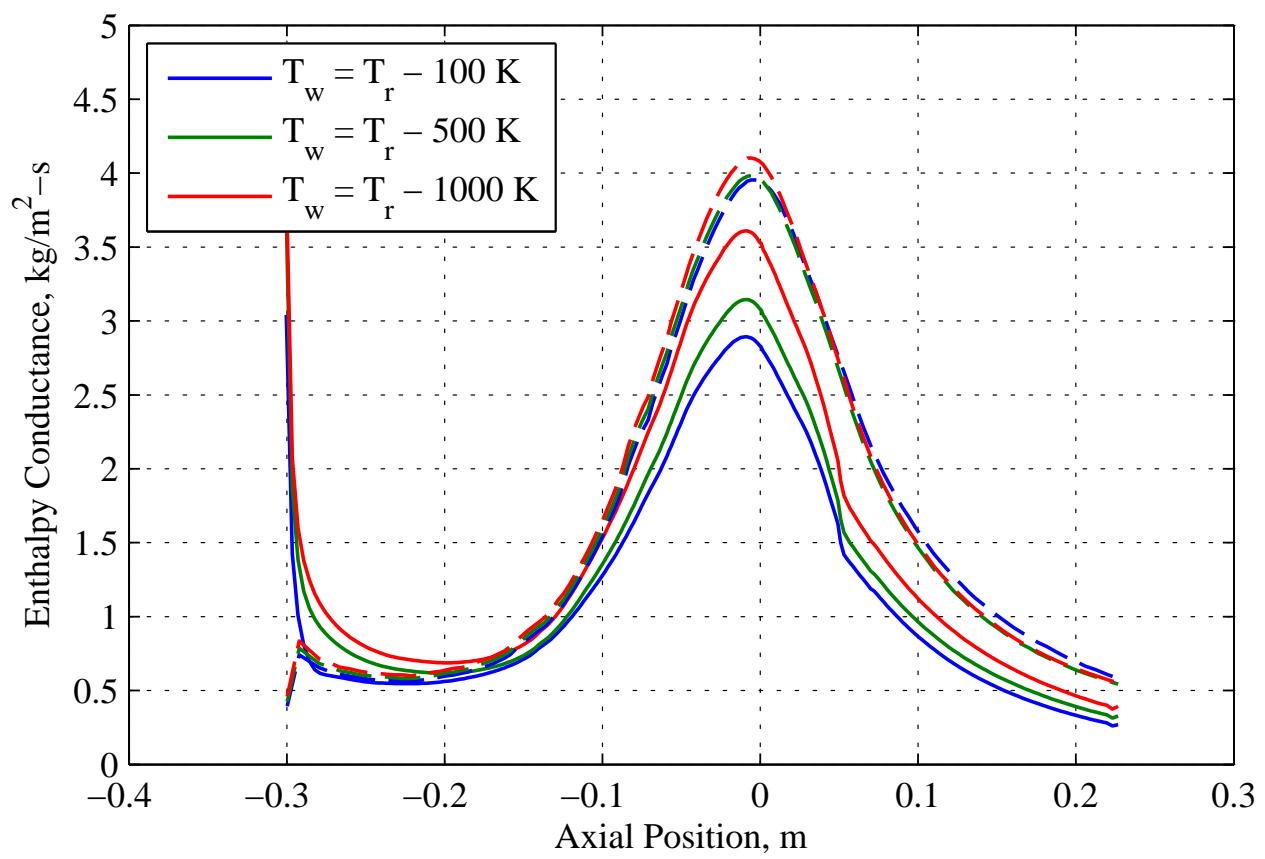

Figure 8. Enthalpy conductance as a function of axial position, for a range of recovery temperature offset values, as computed by LeMANS (solid lines) and NAT (dashed lines). Chamber pressure is $4.48 \mathrm{MPa}$. 
For this work, most material response simulations are computed using convection boundary conditions for the assumption that the wall temperature is offset $1000 \mathrm{~K}$ below the local recovery temperature. Enthalpy conductance as a function of time and axial position in the nozzle is presented in Fig. 9.

\section{IV.C. Results}

Multiple simulations of the HIPPO nozzle are performed using the MOPAR-MD thermal response code. From these simulations, it is possible to make comparisons to determine the effect of different simulation parameters on the ablation response of the nozzle. Parameters considered include the boundary condition computation method, the presence or absence of stream radiation, and the assumed wall temperature used when computing the convective boundary conditions.

\section{IV.C.1. Boundary Condition Comparison}

In this study a comparison is made to determine what impact the choice of boundary condition computation method (NAT or LeMANS) has on the ablation response of the nozzle. The thermal response of the throat surface is compared in Fig. 10, while recession at the throat is compared in Fig. 11. It can be seen that for the simulation using the enthalpy conductance computed with NAT, the throat surface temperature rises more quickly during the initial transient portion of the simulation. After this point there is little difference between the two simulations, suggesting that the code used to compute the boundary conditions has minimal impact on the surface temperature response. However, surface recession is tightly linked to the magnitude of the enthalpy conductance (and therefore strongly affected by the code used to compute the enthalpy conductance). The simulation using LeMANS-computed enthalpy conductance values predicts substantially less surface recession than that using NAT-computed boundary conditions $(0.98 \mathrm{~cm}$ vs. $1.14 \mathrm{~cm}$, or $14 \%$ less). However, this is still significantly greater than the experimentally-measured values of $0.49 \mathrm{~cm}$ to $0.72 \mathrm{~cm}$.

This large discrepancy between the experimentally-measured surface recession and the predicted value could be the result of a number of potential causes. It is possible that the material model used in the analysis does not accurately represent the actual material used in the experiment. Part of the discrepancy could be caused by swell of the material, for which there is some experimental evidence, 36 but is not included in the model. This analysis assumed a unity Lewis number. However, in reality, the Lewis number for a rocket motor is less than one, which means that the mass transport coefficient will actually be less than the heat transport coefficient. This would reduce the extent of the surface recession. The convective boundary conditions used in this analysis are all computed based on the original geometry. However, surface recession obviously modifies the nozzle geometry, which could lead to reduced enthalpy conductance values. Finally, in this analysis equilibrium surface chemistry is assumed; if finite-rate chemistry were to occur at the surface this would lead to reduced surface recession. Many of these factors will be explored in future work when coupled flow-field / ablation simulations are performed.

\section{IV.C.2. Radiation Comparison}

This comparison is made to determine the impact of stream radiation on the thermal response of the nozzle. One simulation includes radiative heat transfer, using the correlation for the stream emissivity as discussed in section IV.B.1; in the second simulation, radiation is neglected. The thermal response of the surface of the throat is plotted in Fig. 12, where it can be seen that including stream radiation increases the throat surface temperature by about $66 \mathrm{~K}$, or about $2.5 \%$. The surface temperature distribution is compared at select instances in time in Fig. 13. Generally, including radiation results in higher surface temperatures, especially upstream of the throat, where the differential can be several hundred degrees. However, during the ramp-down at the end of the simulation, including radiation causes the surface temperature to drop faster than if radiation is neglected.

Surface recession at the throat is compared in Fig. 14. Including stream radiation increases surface recession at the throat by only about $3 \%$. The surface recession distribution is compared at select instances in time in Fig. 15. The presence or absence of radiation has almost no impact on recession downstream of the throat, but does make a noticeable difference in the converging portion of the nozzle.

What these comparisons suggest is that, assuming that the correlation for the stream emissivity is accurate, stream radiation has minimal impact on the thermal response and ablation of the throat or downstream

18 of 27

American Institute of Aeronautics and Astronautics 


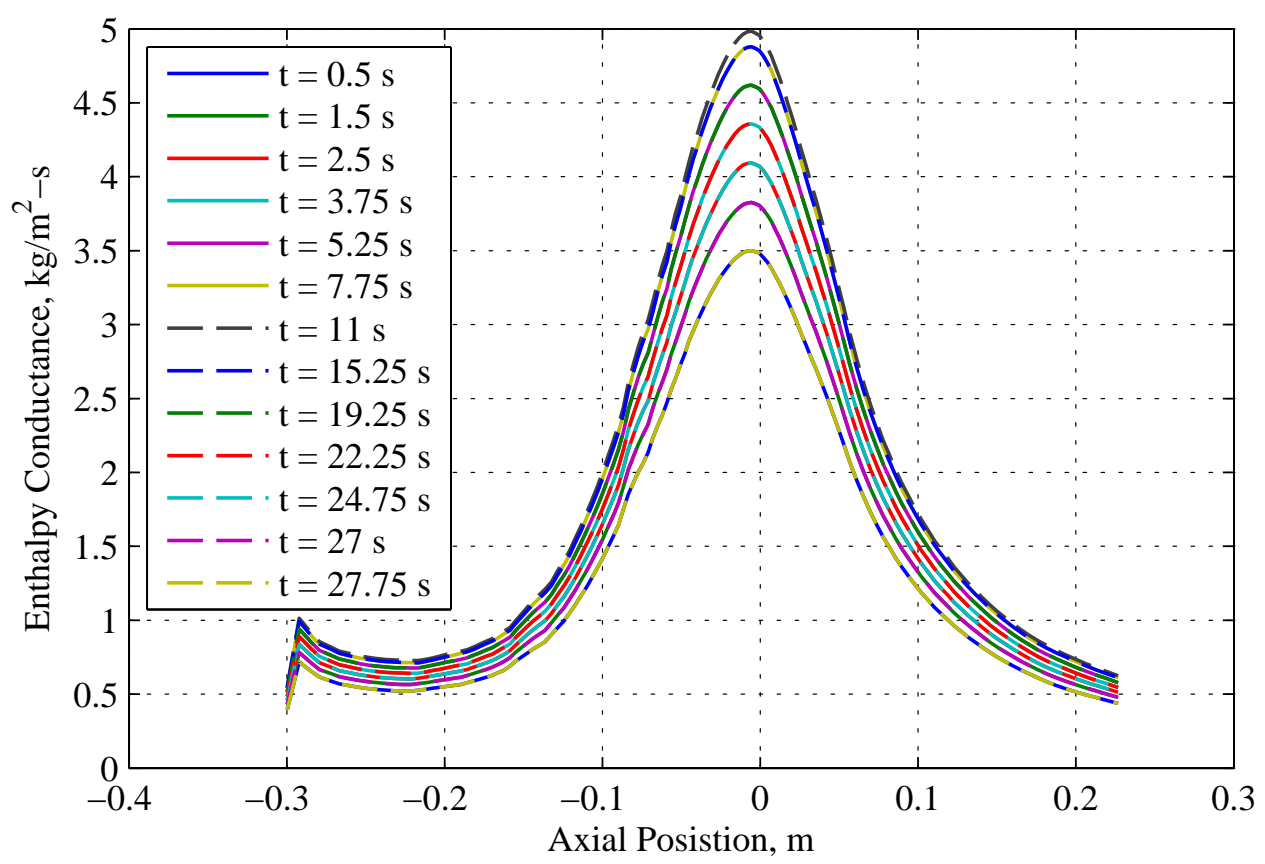

(a) Computed with NAT.

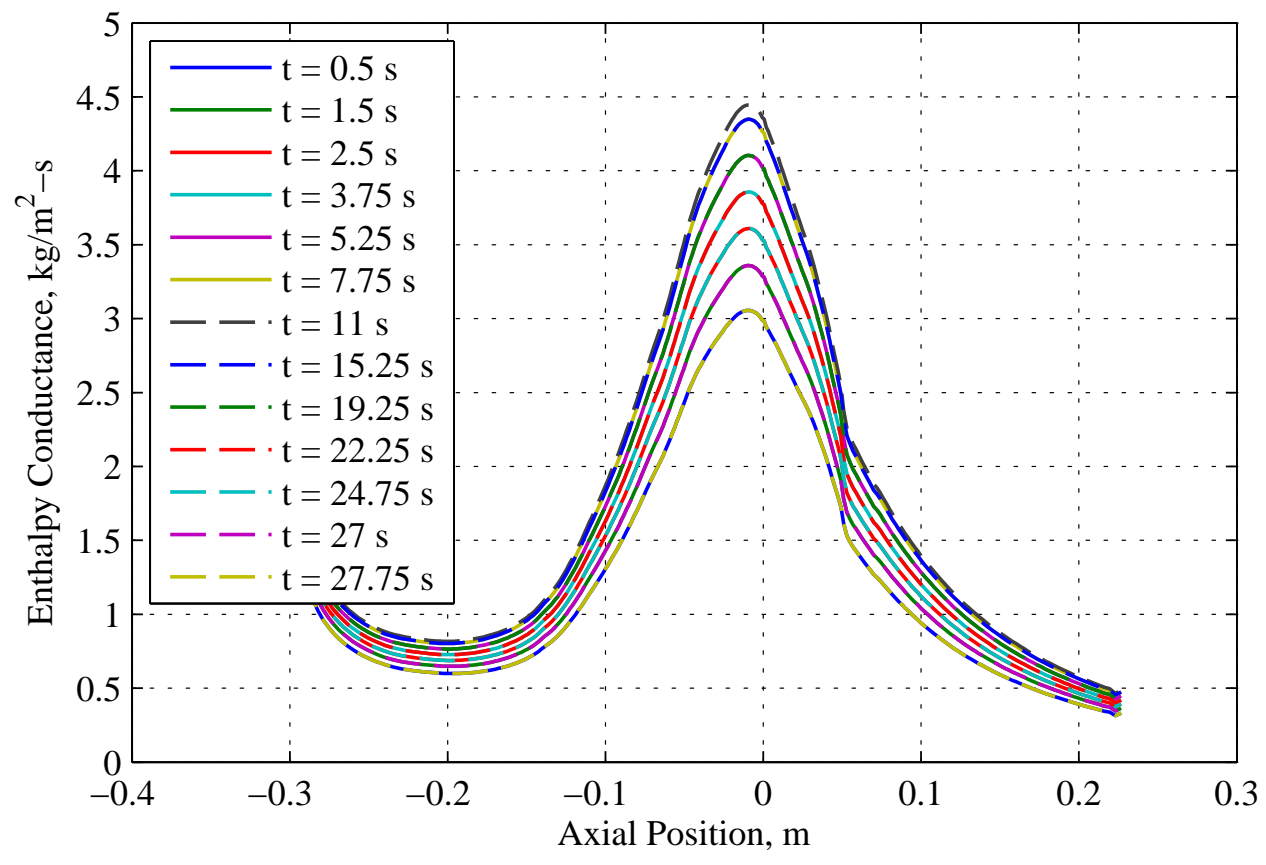

(b) Computed with LeMANS.

Figure 9. Enthalpy conductance on the HIPPO nozzle boundary as a function of axial position and time, for $T_{w}=T_{r}-1000 \mathrm{~K}$. 


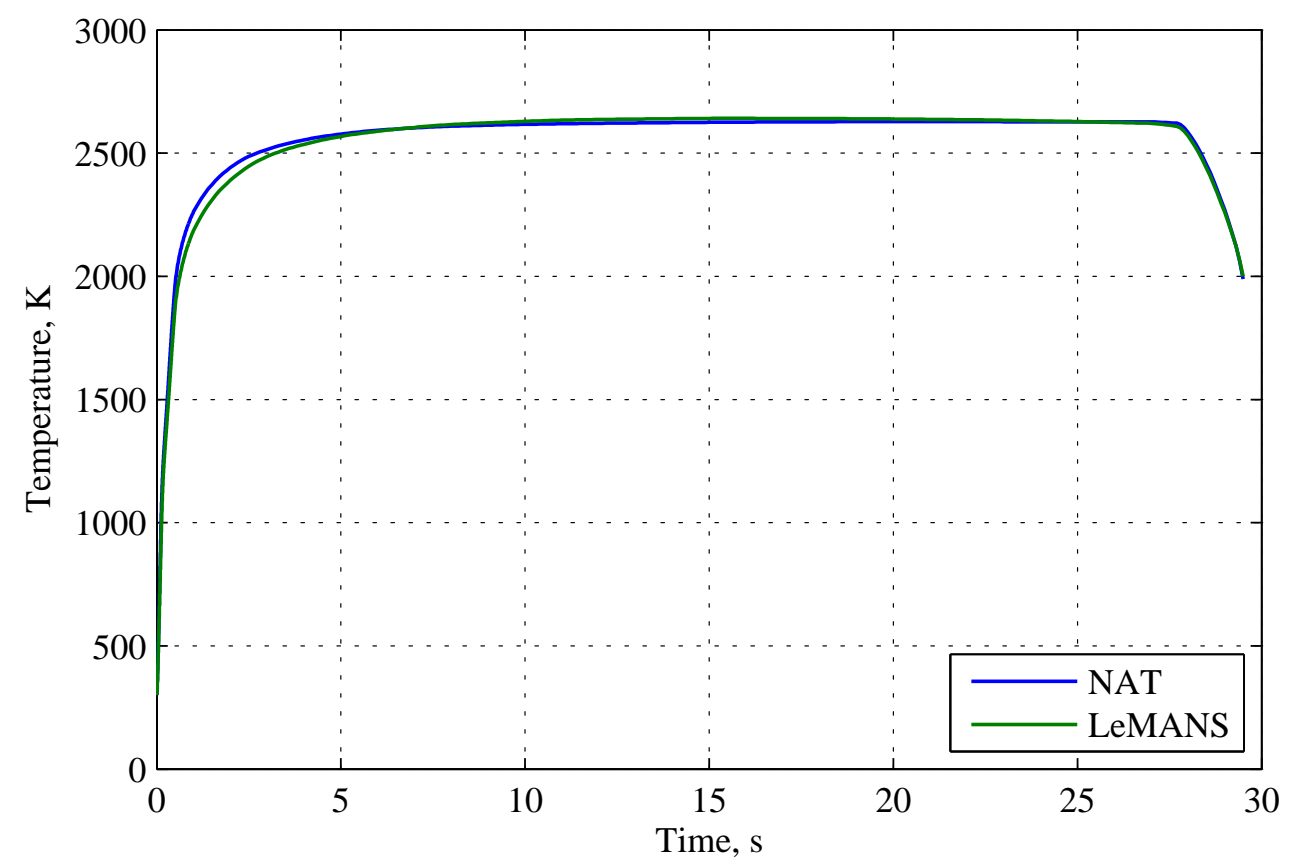

Figure 10. Surface temperature at the throat of the HIPPO nozzle as a function of time, for simulation using enthalpy conductance computed with NAT (blue) and with LeMANS (green).

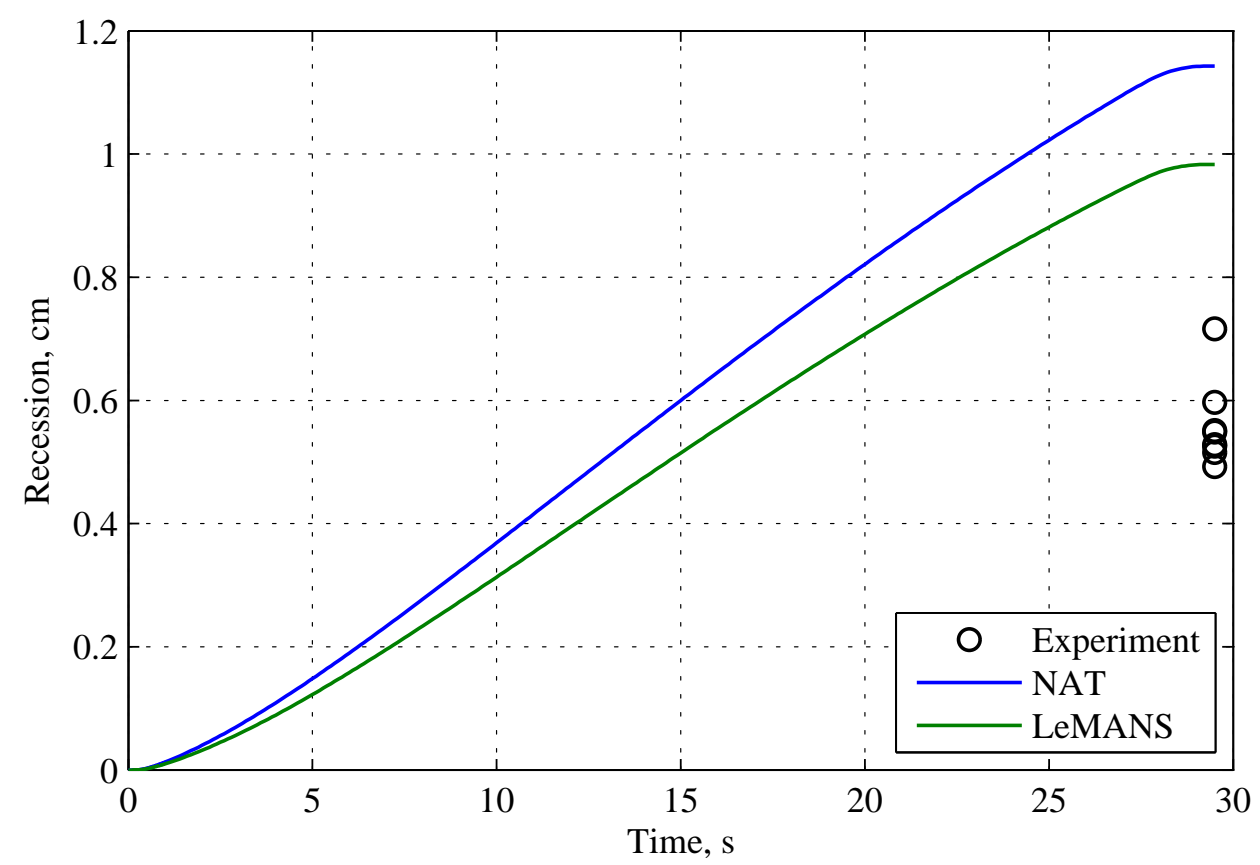

Figure 11. Surface recession at the throat of the HIPPO nozzle as a function of time, for simulation using enthalpy conductance computed with NAT (blue) and with LeMANS (green). Symbols represent final surface recession measured at the conclusion of the experiment. 


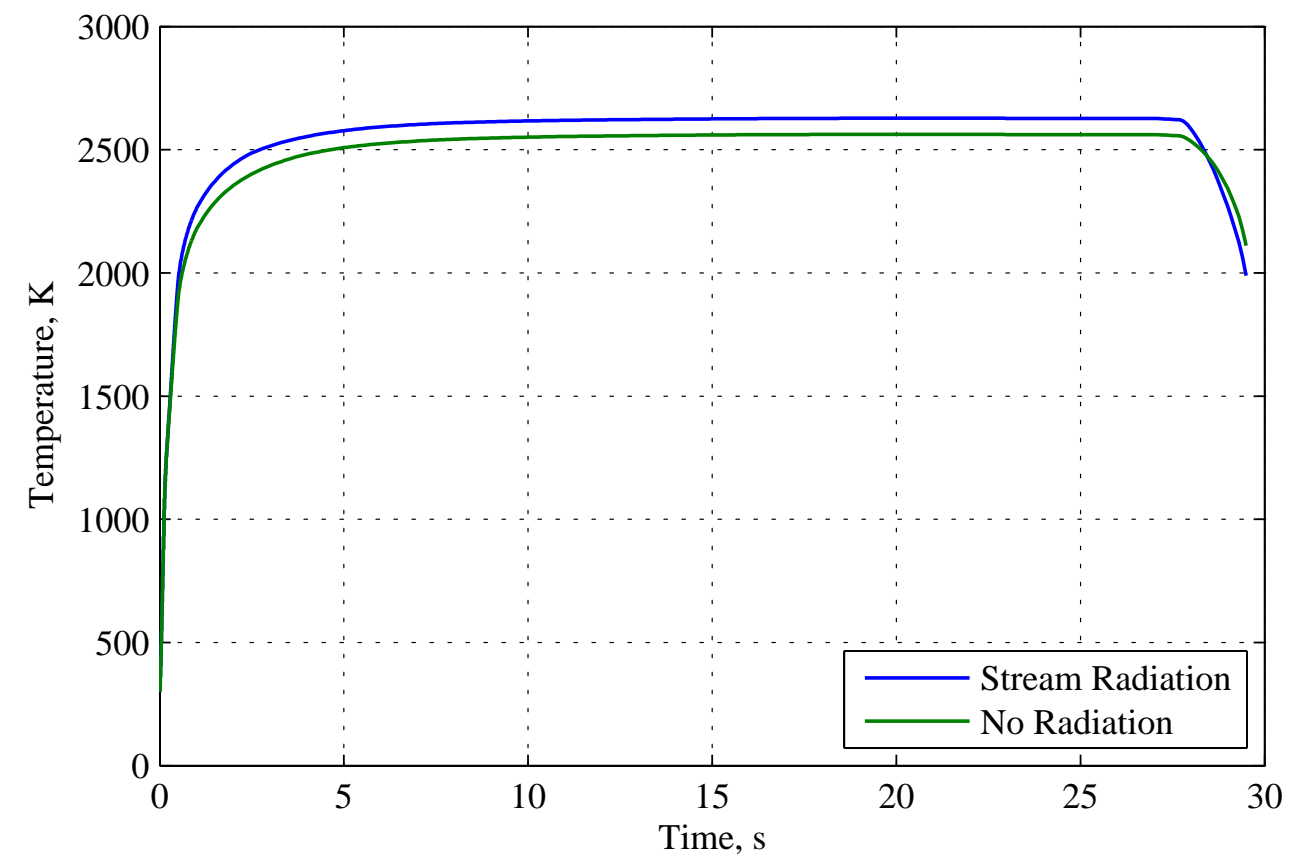

Figure 12. Surface temperature at the throat of the HIPPO nozzle as a function of time, for simulations including radiation (blue) and excluding radiation (green).

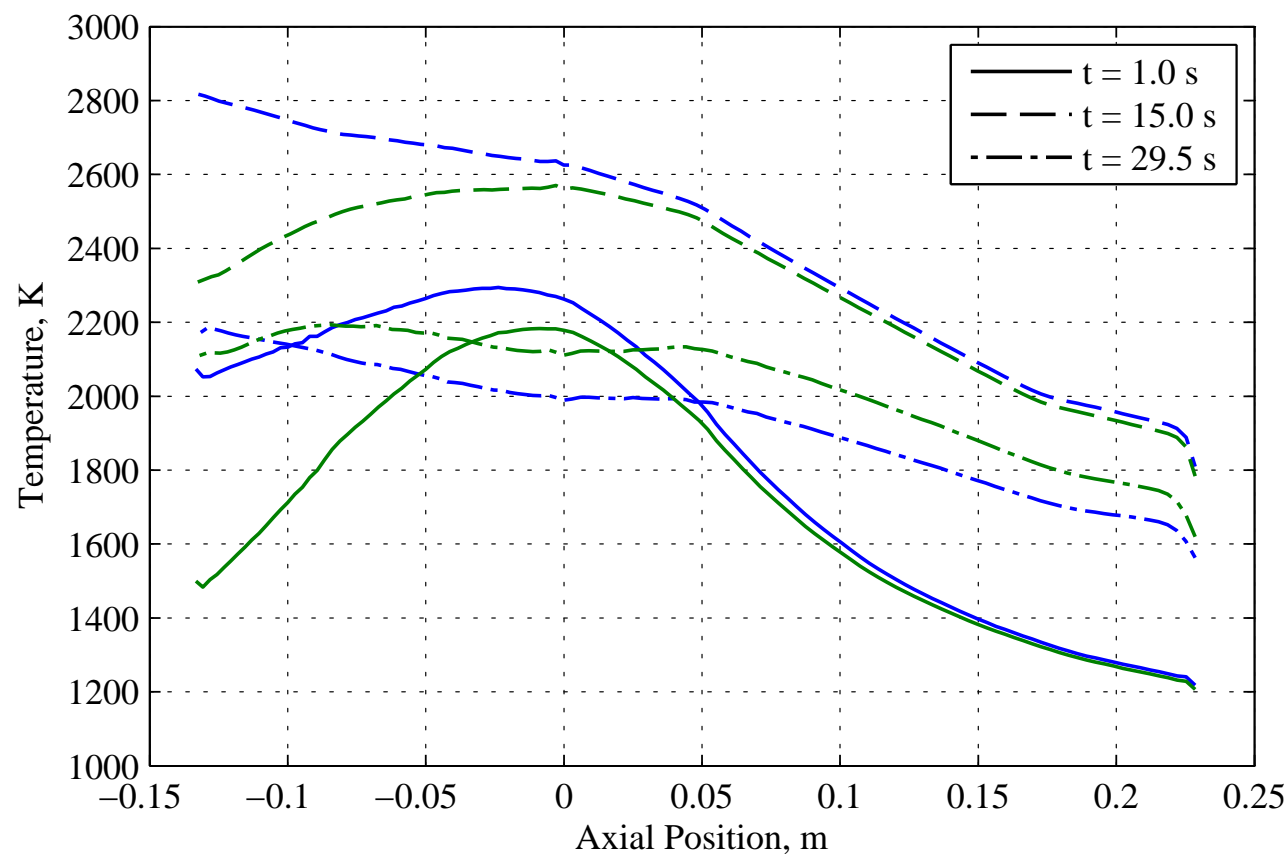

Figure 13. Comparison of surface temperature distribution at select instances in time for the HIPPO nozzle simulation with (blue) and without (green) radiation. 


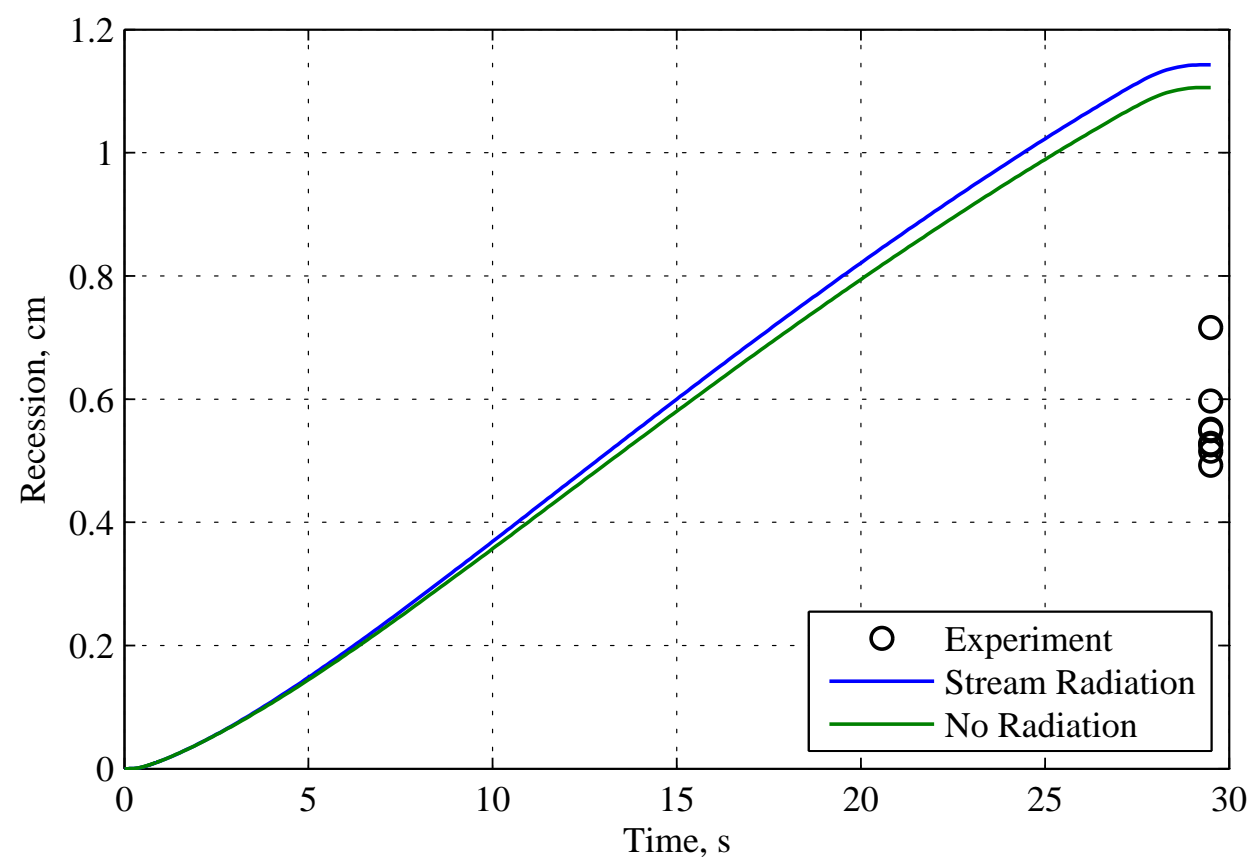

Figure 14. Surface recession at the throat of the HIPPO nozzle as a function of time, for simulation with (blue) and without (green) radiation. Symbols represent final surface recession measured at the conclusion of the experiment.

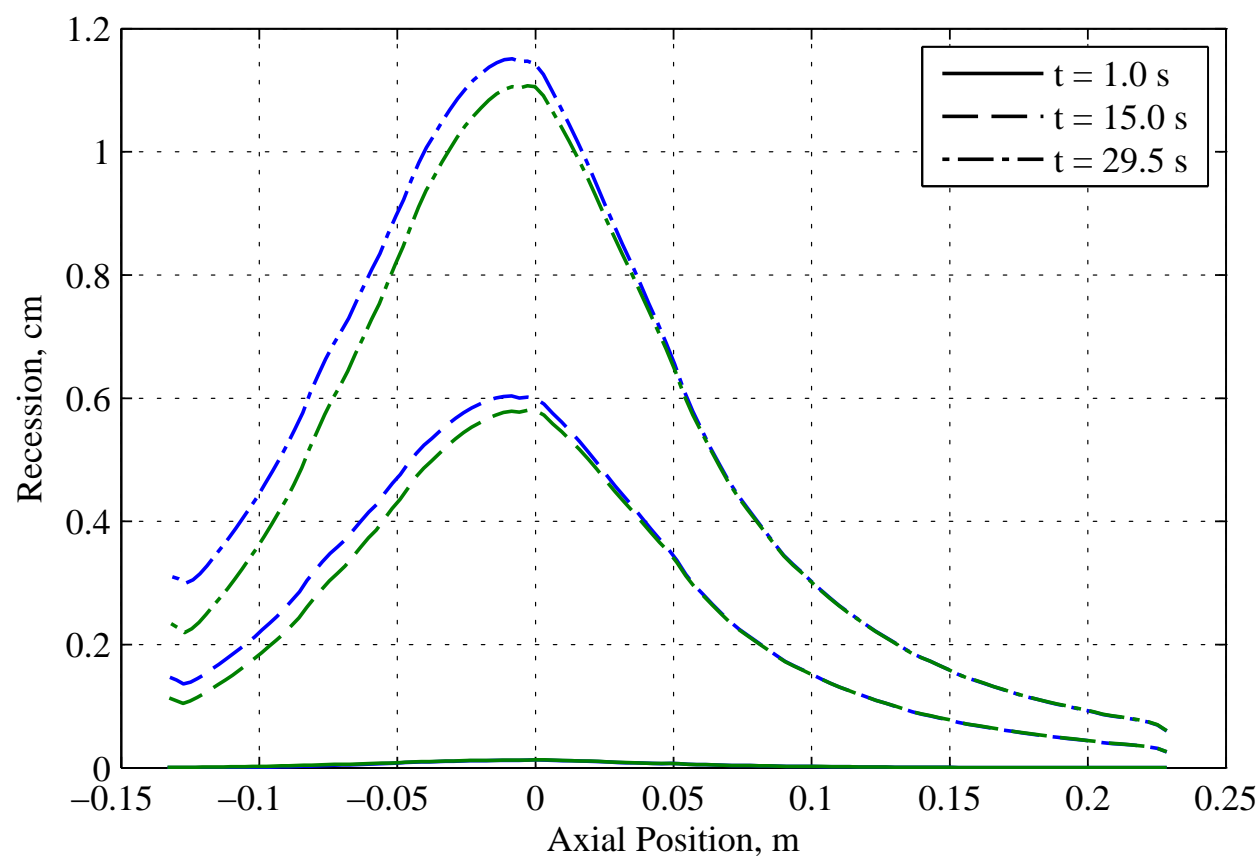

Figure 15. Comparison of surface recession distribution at select instances in time for the HIPPO nozzle simulation with (blue) and without (green) radiation. 
regions of the nozzle. However, it does appear that stream radiation can have a significant effect in the motor chamber and upstream regions of the nozzle, for solid rocket motors with aluminized propellants.

\section{IV.C.3. Wall Temperature Comparison}

This final comparison is made to determine the impact that the assumed wall temperature (when computing the convective boundary conditions) has on the thermal response of the nozzle. Three different temperature profiles are considered. The first profile assumes a wall temperature that is $1000 \mathrm{~K}$ below the local recovery temperature, while the second profile only assumes an offset of $500 \mathrm{~K}$. The third profile is that obtained from a MOPAR-MD simulation of the HIPPO nozzle (at $t=15$ seconds). This third simulation therefore represents a very loosely-coupled approach for determining the interdependence of wall temperature and convective boundary conditions. The three wall temperature profiles are compared in Fig. 16; representative enthalpy conductance profiles are compared in Fig. 17.

The surface temperature profiles from these three simulations are compared for select instances in time in Fig. 18. It is observed that the assumed wall temperature profile used to compute the convective boundary conditions has minimal impact on the surface thermal response at the throat and upstream portions of the nozzle. Downstream of the throat, however, there are some differences. It can also be observed that the first two profiles considered yield quite similar results; the choice of offset value from the local recovery temperature does not greatly affect the thermal response of the nozzle. However, the third wall temperature profile produces a significantly different thermal response in the downstream portion of the nozzle, predicting surface temperatures that are up to about $200 \mathrm{~K}$ greater than predicted based on the first two profiles.

Surface recession profiles are compared at select instances in time in Fig. 19. This plot suggests that the choice of wall temperature profile used when computing the convective boundary conditions has a significant impact on the predicted nozzle ablation (surface recession). The surface recession profiles predicted with the first two temperature profiles have very similar shape, but the magnitude of the recession is greater (by about $15 \%$ at the throat) for the simulation that used an offset of $1000 \mathrm{~K}$ versus the simulation that assumed an offset of $500 \mathrm{~K}$. The third simulation predicted higher surface recession overall, but especially in the downstream portion of the nozzle.

These comparisons indicate that the thermal response of a nozzle is somewhat dependent upon the assumed wall temperature profile used to compute convective boundary conditions, and that the surface recession response of the nozzle is quite sensitive to the assumed profile. These observations support the hypothesis that it is necessary to perform fully-conjugate, coupled flow-field / ablation simulations in order to accurately capture the thermal response and ablation of rocket nozzles.

\section{Conclusion}

A new two-dimensional ablation analysis code (MOPAR-MD) capable of modeling pyrolyzing thermal protection system (TPS) materials has been presented. The mesh deformation, energy, solid phase continuity, and gas phase continuity equations are loosely-coupled and solved sequentially; multiple inner iterations are performed in order to bring all variables into agreement each time step. Materials with anisotropic thermal conductivity and permeability can be modeled, and an arbitrary number of components can be used to model the pyrolysis process. The ablating boundary condition implementation is based upon a unity Lewis number assumption. Code implementation has been thoroughly verified by simulating a sequence of test cases. Favorable agreement with analytical solutions and results from other (one-dimensional) ablation codes indicates a correct implementation consistent with other codes.

This new material response code can be coupled to the LeMANS reacting flow solver. New capabilities required for modeling nozzle flow-fields have been added to LeMANS. The Menter BSL and SST turbulence models were implemented and validated, and shown to yield accurate heat transfer predictions. A "two gas" method was implemented to properly model the thermodynamics of the gas-particle flow found in many rocket nozzles. The flow through a rocket nozzle test case was observed to "freeze", indicating the need for the use of a multidimensional flow solver with a finite-rate chemistry mechanism in order to obtain accurate predictions.

The new MOPAR-MD code was used to predict the thermal and ablation response of the HIPPO nozzle test case. Only uncoupled flow-field / ablation analyses have been performed at this time; future work will focus extensively on performing fully-conjugate, coupled simulations. It was observed that the convective

23 of 27

American Institute of Aeronautics and Astronautics 


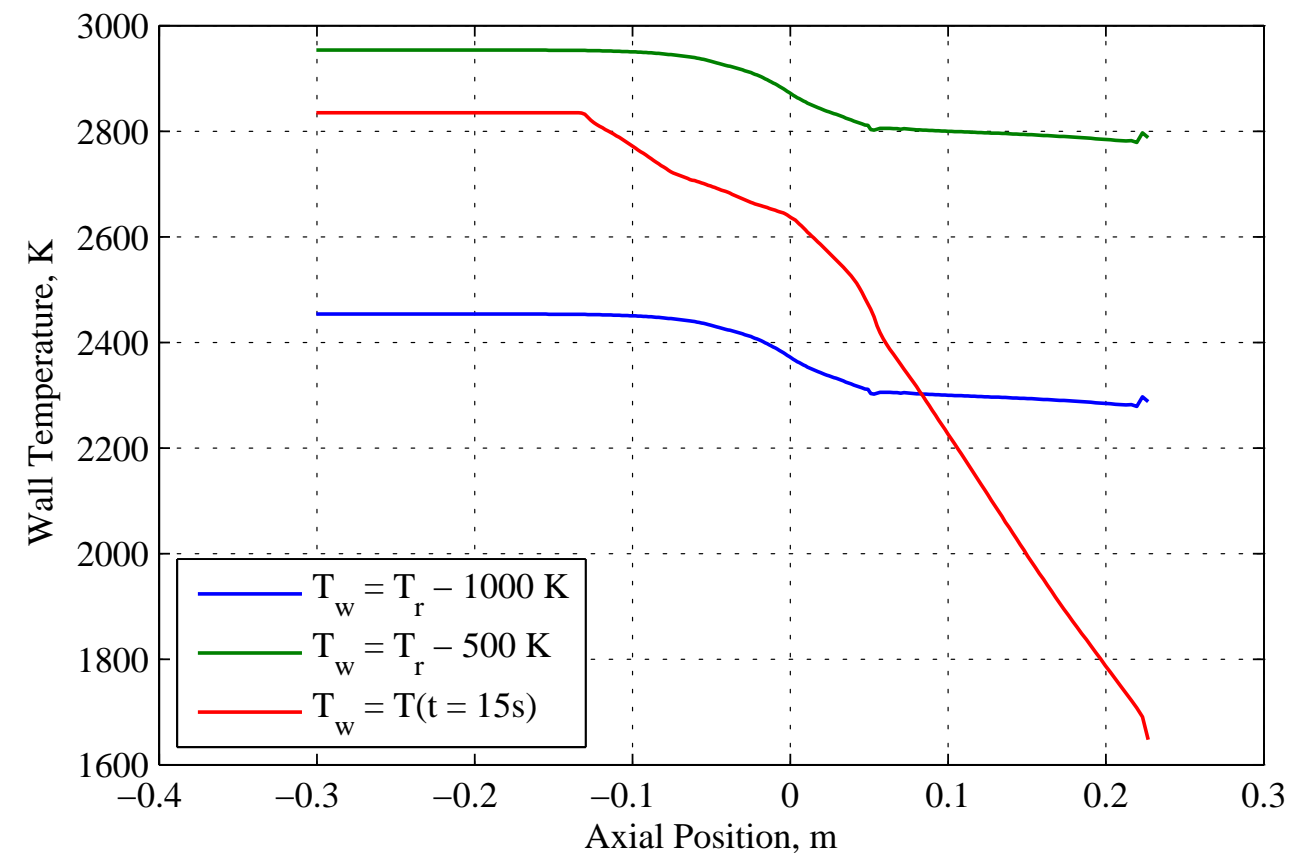

Figure 16. Comparison of the wall temperature profiles used for the wall temperature comparison study.

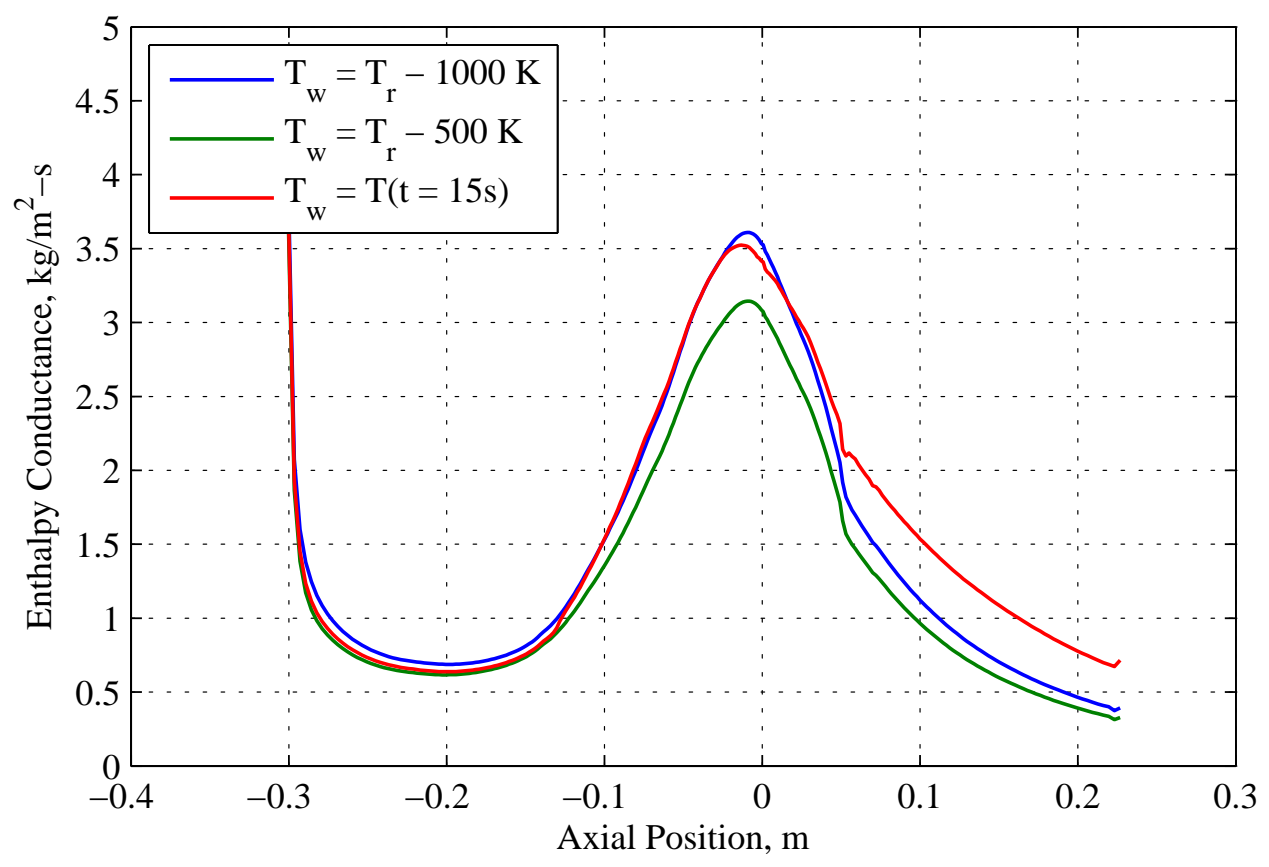

Figure 17. Comparison of enthalpy conductance as computed with LeMANS for the three different assumed wall temperature profiles. Chamber pressure is $4.48 \mathrm{MPa}$. 


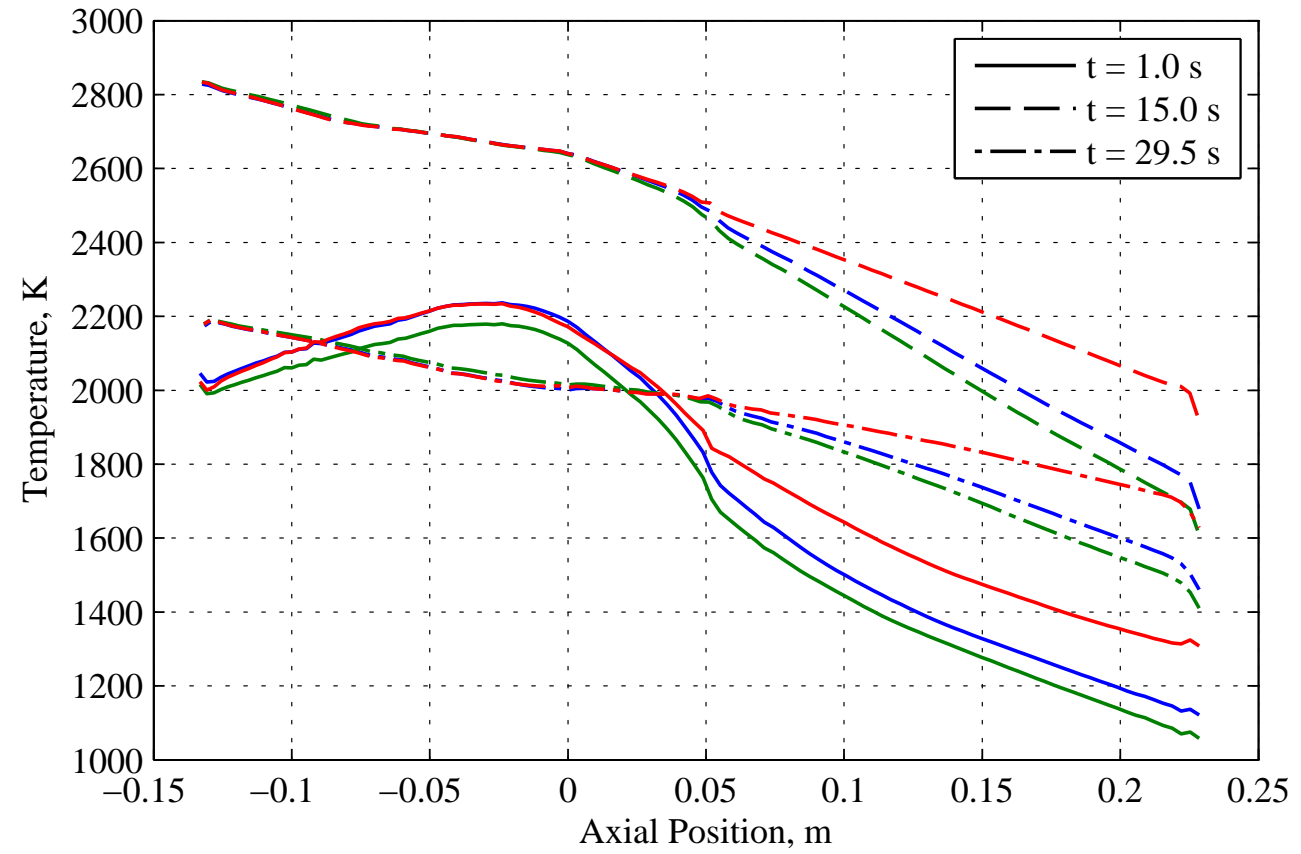

Figure 18. Comparison of surface temperature distribution at select instances in time for the HIPPO nozzle simulation using enthalpy conductance computed with $T_{w}=T_{r}-1000 \mathrm{~K}$ (blue), $T_{w}=T_{r}-500 \mathrm{~K}$ (green), and $T_{w}=T(t=15 \mathrm{~s}$ ) (red).

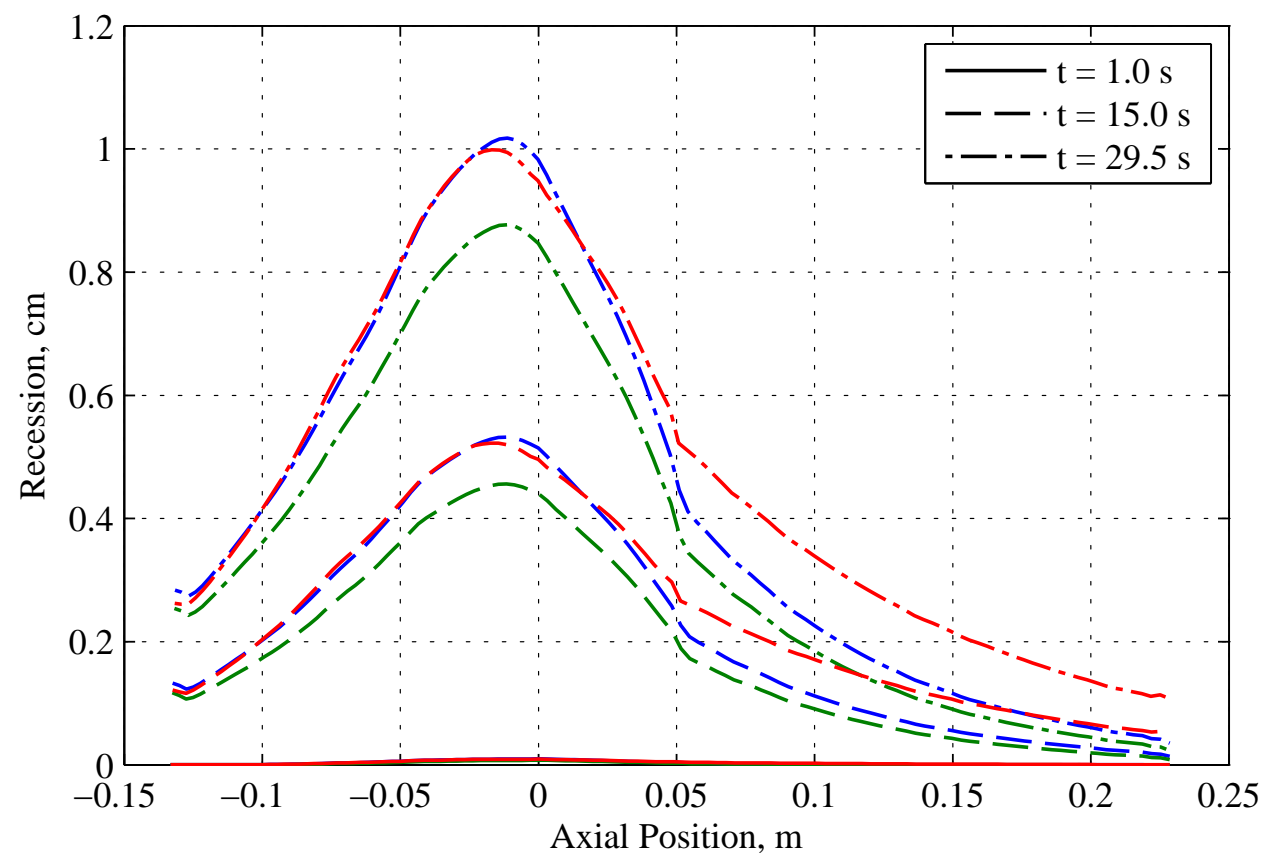

Figure 19. Comparison of surface recession distribution at select instances in time for the HIPPO nozzle simulation using enthalpy conductance computed with $T_{w}=T_{r}-1000 \mathrm{~K}$ (blue), $T_{w}=T_{r}-500 \mathrm{~K}(\mathrm{green})$, and $T_{w}=T(t=15 \mathrm{~s})$ (red). 
heating boundary condition predicted by the LeMANS flow solver is less severe than that predicted by an integral boundary layer solution. Enthalpy conductance was found to be extremely sensitive to the assumed wall temperature, underscoring the need to perform coupled flow-field / ablation simulations in order to most accurately capture the convective heating environment. The radiation exchange between the nozzle wall and the combustion gases was modeled with a commonly-used correlation. Comparisons suggest that radiation has minimal impact on the thermal response and ablation of the throat and downstream portions of the rocket nozzle; radiation is more important, however, in the motor chamber and upstream portions of the nozzle. Other comparisons show that while the thermal response of the nozzle is only somewhat dependent on the assumed wall temperature profile used to compute the convective boundary conditions, the surface recession of the nozzle is quite sensitive. This further supports the hypothesis that it is necessary to perform fully-conjugate, coupled flow-field / ablation simulations in order to accurately predict ablation of rocket nozzles.

All simulations of the HIPPO nozzle predicted substantially greater surface recession than measured experimentally. Several potential factors for this discrepancy have been identified, including the carbon phenolic material model, swelling of the material, the assumption of a unity Lewis number, geometric effects on the convection coefficient, and finite-rate surface chemistry. Many of these factors will be explored in future work when coupled flow-field / ablation simulations are performed.

Future work will focus on performing fully-conjugate, coupled flow-field / ablation simulations of the HIPPO nozzle. In this approach it is expected that it will be possible to accurately capture the effects of the wall temperature on the convective boundary conditions. It will also be possible to account for the effects of surface recession and changing nozzle geometry on the convective heating environment, and the resultant nozzle ablation. It is also planned to relax the equilibrium chemistry, unity Lewis number assumption, and explore the effects of finite-rate surface chemistry and non-unity Lewis number. By basing thermal protection system engineering more firmly on first principles, improved accuracy is anticipated.

\section{Acknowledgments}

Funding for this work was provided by the NAWCWD Fellowship Program, the SMART Scholarship, and NAWCWD IAR 5097. Computational resources were provided in part by the Department of Defense High Performance Computing Modernization Office. Thanks are extended to Dr. Mark Ewing for fruitful conversations and suggestions for running ITRAC.

\section{References}

\footnotetext{
${ }^{1}$ Bianchi, D., Turchi, A., Nasuti, F., and Onofri, M., "Chemical Erosion of Carbon-Phenolic Rocket Nozzles with FiniteRate Surface Chemistry," Journal of Propulsion and Power, June 2013, pp. 1220-1230.

${ }^{2}$ Bianchi, D. and Nasuti, F., "Carbon-Carbon Nozzle Erosion and Shape-Change Effects in Full-Scale Solid-Rocket Motors," Journal of Propulsion and Power, Vol. 28, No. 4, July 2012, pp. 820-830.

${ }^{3}$ Bianchi, D., Nasuti, F., and Martelli, E., "Coupled Analysis of Flow and Surface Ablation in Carbon-Carbon Rocket Nozzles," Journal of Spacecraft and Rockets, Vol. 46, No. 3, May 2009, pp. 492-500.

${ }^{4}$ Alkandry, H., Boyd, I. D., and Martin, A., "Coupled Flow Field Simulations of Charring Ablators with Nonequilibrium Surface Chemistry," 44th AIAA Thermophysics Conference, June 2013, AIAA 2013-2634.

${ }^{5}$ Wiebenga, J. and Boyd, I., "Computation of Multi-Dimensional Material Response Coupled to Hypersonic Flow," 43rd AIAA Thermophysics Conference, June 2012, AIAA 2012-2873.

${ }^{6}$ Martin, A. and Boyd, I., "Strongly coupled computation of material response and nonequilibrium flow for hypersonic ablation," 41st AIAA Thermophysics Conference, June 2009, AIAA 2009-3597.

${ }^{7}$ Wiebenga, J. E., High-Fidelity Material Response Modeling as Part of an Aerothermoelastic Framework for Hypersonic Flows, Ph.D. thesis, University of Michigan, Ann Arbor, MI, 2014.

${ }^{8}$ Scalabrin, L. C., Numerical Simulation of Weakly Ionized Hypersonic Flow Over Reentry Capsules, Ph.D. thesis, University of Michigan, Ann Arbor, MI, 2007.

${ }^{9}$ Amar, A. J., Modeling of One-Dimensional Ablation with Porous Flow Using Finite Control Volume Procedure, Master's thesis, North Carolina State University, Raleigh, NC, 2006.

${ }^{10}$ Amar, A. J., Blackwell, B. F., and Edwards, J. R., "One-Dimensional Ablation Using a Full Newton's Method and Finite Control Volume Procedure," Journal of Thermophysics and Heat Transfer, Vol. 22, No. 1, January 2008, pp. 71-82.

${ }^{11}$ Amar, A. J., Blackwell, B. F., and Edwards, J. R., "Development and Verification of a One-Dimensional Ablation Code Including Pyrolysis Gas Flow," Journal of Thermophysics and Heat Transfer, Vol. 23, No. 1, January 2009, pp. 59-71.

${ }^{12}$ Martin, A. and Boyd, I. D., "Non-Darcian Behavior of Pyrolysis Gas in a Thermal Protection System," Journal of Thermophysics and Heat Transfer, Vol. 24, No. 1, January 2010, pp. 60-68.
} 
${ }^{13}$ Martin, A. and Boyd, I., "Simulation of Pyrolysis Gas Within a Thermal Protection System," 40th Thermophysics Conference, June 2008, AIAA 2008-3805.

${ }^{14}$ Nield, D. A. and Bejan, A., Convection in Porous Media, Springer, 4th ed., 2013.

${ }^{15}$ Baliga, B. R., A Control-Volume Based Finite-Element Method for Convective Heat and Mass Transfer, Ph.D. thesis, University of Minnesota, Minneapolis, MN, 1978.

${ }^{16}$ Voller, V. R., Basic Control Volume Finite Element Methods for Fluids and Solids., World Scientific Publishing Co. Pte. Ltd., 2009.

${ }^{17}$ Blackwell, B. F. and Hogan, R. E., "Numerical Solution of Axisymmetric Heat Conduction Problems Using Finite Control Volume Technique," Journal of Thermophysics and Heat Transfer, Vol. 7, No. 3, January 1993, pp. 462-471.

${ }^{18}$ Hogan, R. E., Blackwell, B. F., and Cochran, R. J., "Application of Moving Grid Control Volume Finite Element Method to Ablation Problems," Journal of Thermophysics and Heat Transfer, Vol. 10, No. 2, April 1996, pp. 312-319.

${ }^{19}$ Chen, Y.-K. and Milos, F. S., "Two-Dimensional Implicit Thermal Response and Ablation Program for Charring Materials," Journal of Spacecraft and Rockets, Vol. 38, No. 4, July 2001, pp. 473-481.

${ }^{20}$ Saad, Y. and Schultz, M. H., "GMRES: A Generalized Minimal Residual Algorithm for Solving Nonsymmetric Linear Systems," SIAM Journal on Scientific and Statistical Computing, Vol. 7, No. 3, 1986, pp. 856-869.

${ }^{21}$ Saad, Y., Iterative Methods for Sparse Linear Systems, SIAM, 2nd ed., 2003.

${ }^{22}$ Acurex Corporation, Mountain View, CA, User's Manual, Non-Proprietary, Aerotherm Charring Material Thermal Response and Ablation Program, CMA87S, November 1987.

${ }^{23}$ Kays, W. M., Crawford, M. E., and Weigand, B., Convective Heat and Mass Transfer, McGraw-Hill, 4th ed., 2005.

${ }^{24}$ Aerotherm Corporation, Mountain View, CA, Aerotherm Chemical Equilibrium (ACE93), July 1993.

${ }^{25}$ Ewing, M. E., Laker, T. S., and Walker, D. T., "Numerical Modeling of Ablation Heat Transfer," Journal of Thermophysics and Heat Transfer, Vol. 27, No. 4, October 2013, pp. 615-632.

${ }^{26}$ Suzuki, T., Sawada, K., Yamada, T., and Inatani, Y., "Gas Permeability of Oblique-Layered Carbon-Cloth Ablator," Journal of Thermophysics and Heat Transfer, Vol. 18, No. 4, October 2004, pp. 548-550.

${ }^{27}$ McManus, H. L. N., High-Temperature Thermomechanical Behavior of Carbon-Phenolic and Carbon-Carbon Composites, Ph.D. thesis, Stanford University, Stanford, CA, 1990.

${ }^{28}$ McManus, H. L. and Springer, G. S., "High Temperature Thermomechanical Behavior of Carbon-Phenolic and CarbonCarbon Composites, II. Results," Journal of Composite Materials, Vol. 26, No. 2, January 1992, pp. $230-255$.

${ }^{29}$ Lachaud, J., Martin, A., Cozmuta, I., and Laub, B., "Ablation Workshop Test Case," 4th Ablation Workshop, March 2011, http://ablation2012.engineering.uky.edu/files/2012/02/Test_Case_1.pdf [cited 23 June 2015].

${ }^{30}$ Lachaud, J., Martin, A., van Eekelen, T., and Cozmuta, I., "Ablation Test-Case Series \#2," 5th Ablation Workshop, March 2012, http://ablation2012.engineering.uky.edu/files/2012/02/Test_Case_2.pdf [cited 23 June 2015].

31 "Theoretical Ablative Composite for Open Testing (TACOT) Ver. 2.2," http://ablation2012.engineering.uky.edu/files/ 2012/02/TACOT_2.2.xls [cited 23 June 2015].

${ }^{32}$ Menter, F. R., "Two-Equation Eddy-Viscosity Turbulence Models for Engineering Applications," AIAA Journal, Vol. 32, No. 8, August 1994, pp. 1598-1605.

${ }^{33}$ Tong, X.-L., Luke, E., and Tang, L., "Evaluation of the Shear-Stress Transport Turbulence Model for Heat Transfer Applications," 41st Aerospace Sciences Meeting and Exhibit, January 2003, AIAA 2003-0769.

${ }^{34}$ Tong, X.-L. and Luke, E., "Turbulence Models and Heat Transfer in Nozzle Flows," AIAA Journal, Vol. 42, No. 11, November 2004, pp. 2391-2393.

${ }^{35}$ Suzen, Y. and Hoffmann, K., "Performance Study of Turbulence Models for Heat Transfer Predictions," 32nd Thermophysics Conference, June 1997, AIAA 1997-2568.

${ }^{36}$ Arnold, J., Dodson, J., and Laub, B., "Subscale Solid Motor Nozzle Tests - Phase IV and Nozzle Materials Screening and Thermal Characterization - Phase V," NASA CR 161254, NASA, June 1979.

${ }^{37}$ Rudinger, G., Fundamentals of Gas-Particle Flow, Elsevier Scientific Publishing Company, 1980.

${ }^{38}$ Kolozsi, J. J., An Investigation of Heat Transfer through the Turbulent Boundary Layer in an Axially Symmetric, Convergent-Divergent Nozzle, Master's thesis, Ohio State University, Columbus, OH, 1958.

${ }^{39} \mathrm{McBride}, \mathrm{B}$. J. and Gordon, S., "Computer Program for Calculation of Complex Chemical Equilibrium Compositions and Applications. Part 2: User's Manual and Program Description," NASA RP-1311-P2, NASA, June 1996.

${ }^{40}$ Troyes, J., Dubois, I., Borie, V., and Boischot, A., "Multi-Phase Reactive Numerical Simulations of a Model Solid Rocket Exhaust Jet," 42nd AIAA/ASME/SAE/ASEE Joint Propulsion Conference \& Exhibit, July 2006, AIAA $2003-4414$.

${ }^{41}$ Schaefer, J. W., Dahm, T. J., Rodriguez, D. A., Reese, Jr., J. J., and Wool, M. R., "Studies of Ablative Material Performance for Solid Rocket Nozzle Applications," NASA CR-72429, March 1968.

${ }^{42}$ Aerotherm Corporation, Huntsville, AL, User's Manual, Nozzle Aero Thermochemistry (NAT) Computer Code, Version 2.0, February 1994.

${ }^{43}$ Pearce, B. E., "Radiative Heat Transfer within a Solid-Propellant Rocket Motor," Journal of Spacecraft and Rockets, Vol. 15, No. 2, March 1978, pp. 125-128. 